OPEN ACCESS

Citation: Trezza D., Punziano G., C.C.DeFalco (2021) Mappareil racconto, raccontare l'emergenza. Voci digitali dai territori, in "Cambio. Rivista sulle trasformazioni sociali», Vol. 11, n. 21: 163-184. doi: 10.36253/cambio-10255

Copyright: (C) 2021 Trezza D., Punziano G., C.C. De Falco. This is an open access, peer-reviewed article published by Firenze University Press (http:// www.fupress.com/cambio) and distributed under the terms of the Creative Commons Attribution License, which permits unrestricted use, distribution, and reproduction in any medium, provided the original author and source are credited.

Data Availability Statement: All relevant data are within the paper and its Supporting Information files.

Competing Interests: The Author(s) declare(s) no conflict of interest.
Open Essays and Researches

\section{Mappare il racconto, raccontare l'emergenza. Voci digitali dai territori ${ }^{1}$}

\author{
Domenico Trezza, Gabriella Punziano, Ciro Clemente De Falco
}

Università degli Studi di Napoli Federico II

domenico.trezza@unina.it

Abstract. Related to the first period of the COVID-19 pandemic (March-June 2020), some recent studies on the application of content analysis of geolocalized tweets (Bashar et alii 2020, Punziano et alii 2020) have demonstrated the negative relation between the Coronavirus spread (with Northern Italy most affected) and the polarity of social narratives about the pandemic. In brief, the «resilient» social narrative of the most impacted regions has corresponded to a negative and worried emergency narrative of the less affected regions. In relation to epidemiological data, the second phase of the pandemic (also referred to as the «second wave», in autumn 2020) has been very different from the first (ISSa 2020). The severity of emergency, without considering questions about the reliability of the first wave data (Istat 2020a), has been more relevant and homogeneous across Italy. The study questions whether there are differences between the geography of contagion and that of the narrative. Given the increasingly homogeneous spread of the virus, the assumption has been that the digital arena also has ended up showing a narrative more united on negative sentiments. The issue is addressed by analyzing a corpus of geolocalized tweets, extracted in the period from the new October lockdown to the partial and fragmented pre-Christmas reopenings in 2020. Following the application of a model combining text mining and GIS analysis, the most recurrent themes in social discourse on Twitter were mapped. This geography of emerging social narratives (COVID-Issues) compared with the geography of contagion spread (COVID-Spread) and the norms (COVID-Measures) allowed to detect the trend in the relationship of these three dimensions during the second emergence from COVID-19.

Keywords: spatial analysis, COVID-measures, COVID-spread, COVID-issues, twittersphere, COVID-19.

\footnotetext{
${ }^{1}$ Il paper è frutto di un lavoro comune. Tuttavia, Domenico Trezza ha curato paragrafi 1, 3 e 5. Gabriella Punziano il paragrafo 4, e Ciro Clemente De Falco i paragrafi 2 e 6 e l'elaborazione delle cartografie.
} 


\section{INTRODUZIONE. UN'EMERGENZA FRAMMENTATA}

L'emergenza da COVID-19 ha catalizzato buona parte dell'attenzione della ricerca nelle scienze umane e sociali. La pandemia ha infatti determinato uno shock non soltanto sanitario, ma anche sociale: sono cambiate radicalmente le abitudini delle persone, e comprendere come quest'evento stia condizionando il modo di vivere e di pensare degli individui e dei gruppi sociali rappresenta sicuramente una sfida quanto mai necessaria. La dimensione, l'estensione e gli impatti che questo fenomeno ha avuto non sono però stati gli stessi sul territorio italiano, in termini epidemiologici (contagi, ricoveri e decessi), economici e sociali. La narrazione mediatica dell'emergenza ha tuttavia preferito porre sullo sfondo queste differenze, accomunando gli italiani in un unico grande spirito di resistenza alle avversità della pandemia (Battistelli e Galatino 2020). In realtà, la prima ondata dell'emergenza ha mostrato una situazione di assoluta frammentarietà. Non solo relativamente alle note differenze Nord-Sud sull'impatto dell'infezione (ISSb 2020), ma anche in relazione alla rappresentazione sociale dell'emergenza. In uno studio condotto da chi scrive nel corso della prima fase della pandemia è stato messo in luce che la narrazione degli italiani sui social è stata diversa a seconda dell'area geografica di residenza, ed addirittura - in alcuni casi - in controtendenza rispetto alla condizione epidemiologica del rispettivo territorio (Punziano et alii 2020). Peraltro, essa è sembrata seguire evoluzioni differenti a seconda delle varie fasi normative che si sono susseguite generando umori, opinioni e reazioni emotive diverse. Tali fasi normative, lo ricordiamo, sono state uguali per tutte le regioni italiane seppure abbiano sortito effetti divergenti sulla componente narrativo-emotiva indagata. Nonostante lo scenario del contagio fosse evidentemente disuguale sul territorio italiano, il Governo ha, infatti, scelto di adottare una linea unica per tutte le regioni, lasciando alle amministrazioni regionali (ed eventualmente a quelle comunali) la facoltà di intervenire a livello locale, facoltà di cui i governatori regionali si sono avvalsi in maniera estremamente residuale demandando al Governo centrale il protagonismo nella gestione normativa dell'emergenza sociosanitaria nazionale. L'ondata autunnale del virus ha visto ridotte le diversità territoriali nell'andamento di diffusione dei contagi, attenuando le differenze tra Nord e Sud registrate nel corso della prima ondata. Le misure di contenimento adottate dal Governo questa volta però non sono state unitarie, ma hanno raggruppato le regioni in scenari a seconda di tre livelli di rischio differenti identificate dai colori «giallo», «arancione»e «rosso» (DPCM 3 Novembre 2020), qui definibili come «geografie delle misure di contrasto al virus». Quest'intervento normativo ha prodotto una sorta di paradosso rispetto alla prima ondata, nella misura in cui con un contesto epidemiologico più omogeneo rispetto alla prima fase della pandemia si è deciso, invece, di agire in maniera differenziata nell'interesse della salvaguardia nazionale dell'economia che avrebbe potuto subire enormi danni da una nuova stretta generalizzata su spostamenti, attività e produzioni a livello nazionale. L'elemento territoriale si è quindi configurato come uno dei fattori centrali nella pianificazione delle strategie di contrasto e contenimento del virus tanto è vero che molti lavori pubblicati durante questa seconda fase hanno considerato il territorio come uno dei fattori chiave per spiegare gli effetti sociali della pandemia, giungendo a tipizzazioni molto efficaci di profili di percezione legate all'area geografica (cfr. Mazzara et alii 2020). A partire dallo scenario descritto sia a livello di contestualizzazione del fenomeno, sia rispetto alle modalità di riflessione emerse intorno ad esso, nello studio che si presenta il territorio, o meglio, l'informazione geografica, risulta decisiva per approfondire quella che chi scrive ha definito «la geografia delle narrazioni» (Punziano et alii 2020: 304) elaborata in un'ottica di ricerca esclusivamente «digital methods» (Rogers 2013). Questa applicazione consente di restituire un quadro multiforme di opinioni, sentimenti ed emozioni che hanno caratterizzato la quotidianità degli italiani durante il periodo emergenziale indagato prendendo ad oggetto le narrazioni social che ne possono essere ricostruite. La ricerca, dunque, prende in esame un corpus di tweet sull'emergenza durante l'autunno 2020 realizzando un'analisi spaziale del contenuto nella Twitter-sfera provando a mettere in luce le diversità territoriali del «racconto digitale» (Meschini 2020), in relazione alle differenti applicazioni locali delle misure anti-COVID che possono essere riassunte nelle «geografie delle misure di contrasto al virus». L'ampia fascia temporale del nostro corpus ci consente di tenere conto di tre momenti di variazione rilevanti che possono essere intervenuti sull'eventuale modifica del discorso social e che corrispondono a tre interventi normativi di rilievo. Il primo è relativo al DPCM del 13 ottobre con il ritorno delle limitazioni generali per il contenimento del contagio. Il secondo, a partire dal DPCM del 3 novembre introduce le differenziazioni regionali attraverso gli scenari 
cromatici di attenzione. Questo è centrale per il nostro discorso, perché riteniamo che l'applicazione diversificata sul territorio delle disposizioni anti-contagio enfatizzi ancora di più le differenze nel sentiment verso la pandemia. Il terzo momento (DPCM 4 dicembre 2020), che mantiene invariate le disposizioni precedenti, è relativo ai divieti in vigore per le feste natalizie che reintroducono una serie di norme unitarie per tutto il Paese. Il contributo si articola in tre parti. La prima riporta una ricostruzione della riflessione condotta nelle scienze sociali in merito a spazialità e narrazioni della pandemia a mezzo social. La seconda presenta la ricostruzione del percorso di metodo seguito e le fasi di analisi condotte. La terza è usato per la presentazione dei risultati e le riflessioni che possono essere desunte rispetto all'importanza della componente spaziale nell'analisi di fenomeni che, per quanto possano trovare una naturale trasposizione sullo scenario digitale, mantengono un legame innegabile con la componente di vissuto fisico e normativo nella sua evoluzione territoriale.

\section{LE SCIENZE SOCIALI E LA PANDEMIA. PERCORSI DI RICERCA INNOVATIVI}

Assimilabile ad un «fatto sociale totale» (Mauss 1925) la pandemia da COVID-19 e le misure intraprese dai governi per contrastarla, hanno avuto un impatto multilivello sulla società e sulle dimensioni sociale, politica, economica, normativa, prima ancora che sanitarie. Numerosi sono stati i lavori di ricerca finalizzati a cogliere i diversi aspetti su cui la pandemia ha impattato in modo diretto e indiretto. Buona parte delle ricerche riguarda chiaramente l'area medica, ma va sottolineato che anche nelle scienze sociali c'è stata una produzione non irrilevante sul tema caratterizzata tanto da riflessioni teoriche quanto da esperienze empiriche. Un'analisi condotta su Scopus rivela che le scienze sociali con 7.957 prodotti scientifici rappresentano la terza area con il maggior numero di pubblicazioni sul tema COVID-19 dopo l'aria medica (58.924) che ovviamente raccoglie buona parte delle pubblicazioni, e l'area della Biochimica e della Genetica (8.734) (cfr. fig. 1).

L'Italia, in quanto fra i primi paesi ad essere colpito dalla diffusione della pandemia e a essersi autoregolamentata attraverso misure restrittive, ha suscitato, come a breve vedremo, un discreto interesse fra i ricercatori dell'area delle scienze sociali. Gli aspetti indagati sono stati molteplici e per lo più associati all'indagine di quali effetti ha avuto la pandemia ed il successivo lockdown sulla società italiana. Gli studi sui flussi di informazione e comunicazione hanno evidenziato che il COVID-19, in quanto prima pandemia globale su vasta scala dell'era digitale, ha presentato nuove sfide al governo italiano, soprattutto per quanto riguarda la diffusione di notizie e la disinformazione. Non a caso la comunicazione delle informazioni riguardanti il rischio di contagio risultata essere stata spesso inefficace, spianando la strada alle "fake news" (Moscadelli et alii 2020) e facendo emergere l'importanza di indagare il ruolo delle fonti di informazione nell'influenzare l'opinione pubblica e la scelta dei cittadini italiani di fidarsi e quindi affidarsi alle istituzioni, accettando le misure necessarie e sostenendo i cambiamenti comportamentali (Falcone e Sapienza 2020; Graffigna et alii 2020). Anche il mondo dell'educazione, che ha

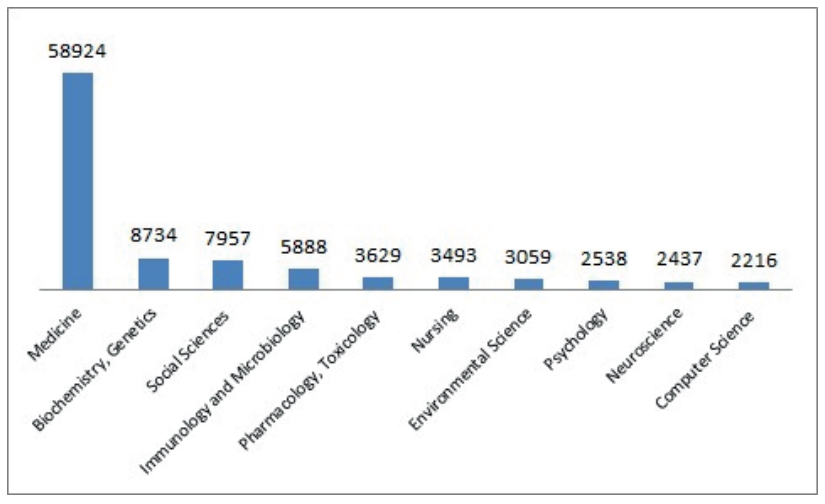

Figura 1. Numero di pubblicazioni per area scientifica (prime 10). Fonte: Scopus. subito notevoli trasformazioni durante la fase della pandemia, ha suscitato molto interesse. Molti autori, infatti, hanno analizzato le nuove sfide per gli studenti a partire dai livelli elementari a quelli universitari (Consiglio et alii 2020) o anche quale sia stato il ruolo delle risorse individuali e delle comunità accademiche nell'affrontare il COVID-19, considerando le limitazioni e i cambiamenti che questo ha comportato (Capone et alii 2020; Procentese et alii 2020; Gaggi et alii 2020). Altri studi hanno indagato le tecnologie più adottate, le metodologie didattiche, nonché l'impatto sulla popolazione scolastica (Giancola e Piromalli 2020; Balzola 2020; 
Cerrocchi 2020). Con riferimento agli effetti sugli individui possono essere richiamate diverse analisi sull'impatto che hanno avuto le misure introdotte - lockdown in primis - per arginare la diffusione della nuova malattia da Coronavirus, sulle condizioni socioeconomiche dei cittadini italiani. Queste evidenziano come la pandemia abbia avuto effetti differenziati in base alla categoria lavorativa d'appartenenza del soggetto (Figari et alii 2020) e di come le nuove modalità di lavoro da casa potrebbero creare disparità in base a genere, età e titolo di studio (Bonacini $e t$ alii 2020). Con riferimento ai settori lavorativi maggiormente colpiti, alcuni studiosi hanno esplorato le intenzioni turistiche e il ruolo della finanza pubblica nel sostenere la domanda di servizi turistici in hotel e B\&B, calata a causa del coronavirus (Istat 2020b). Sono stati oggetto d'attenzione anche gli effetti psicologici (come cambiamenti nei livelli di depressione, stress e ansia) e i fattori associati a questi cambiamenti (Roma et alii 2020; Germani et alii 2020; Lenzo et alii 2020) riscontrati tra gli italiani durante il periodo di quarantena. Ad esempio, secondo Lenzo (2020) molti giovani e adulti sono stati oggetto di forte stress psicologico mostrando preoccupazioni sul loro ruolo come possibili portatori asintomatici di COVID-19 all'interno dei propri nuclei familiari. Famiglie che secondo alcuni studiosi si sono trovate a dover affrontare, oltre alla paura del contagio, numerose e nuove sfide adottando cambiamenti significativi nella routine quotidiana con importanti implicazioni per lo stress familiare (Cusinato $e t$ alii 2020). Con riferimento alle relazioni sociali troviamo studi che si sono concentrati sull'analisi di come siano stati utilizzati durante il periodo di lockdown i siti di social network e di come hanno influenzato la qualità, in particolare, alcuni studiosi, hanno raccolto informazioni sull'utilizzo dei siti di social network da parte degli anziani e come questo potesse riverberarsi sulla solitudine auto-percepita e sull'impegno sociale con la famiglia e gli amici (Rolandi et alii 2020). Oltre che mezzo di relazioni sociali, i social network sono diventati il mezzo di informazione prevalente, sui quali, specialmente durante il lockdown, i maggiori temi di attualità sono stati discussi (De Santis et alii 2020). La pandemia è stata dunque uno dei principali argomenti sui social media e fra questi Twitter, piattaforma utilizzata anche dagli enti istituzionali per le comunicazioni relative alla pandemia (Carelli e Vittadini 2020; Drylie-Carey et alii 2020). Le analisi condotte su questo social hanno permesso di conoscere le reazioni delle persone in relazione alla diffusione nel mondo del COVID-19 (Noor et alii 2020). In particolare, l'utilizzo delle informazioni geografiche derivanti dai social media (Campagna et alii 2016) e quindi dei tweet con il tag geografico ha consentito di indagare l'estensione del dibattito e le sue diverse declinazioni spazio-temporali. Dalle ricerche basate sui tweet geolocalizzati relativi alla pandemia, infatti, emergono sia differenze fra i singoli paesi (Saire Chire e Cruz Oblitas 2020; Suratnoaji et alii 2020; De Santis et alii 2020; Essam e Abdo 2020) sia fra le diverse fasi dell'emergenza così come evidenziato da Chang et alii (2021), che hanno tracciato l'evolversi dei topic più salienti durante i mesi della crisi. Recuperare la dimensione ecologica all'interno di queste ricerche sulla pandemia si è rilevato finora fruttuoso e pertanto, sarà interessante provare ad applicare questa impostazione anche al caso italiano dove si riscontrano pochi tentativi in tal senso (Rivieccio et alii, 2020; De Santis et alii, 2021). In termini più generali si può notare, poi, come di recente lo spazio e le sue caratteristiche - centrali nel lavoro di autori classici come Durkheim (Halbwachs 1918) e Park (Manella 2009) - sebbene inizialmente assenti negli internet o digital studies stiano diventando oggetto d'attenzione crescente nelle analisi incentrate sui social media. Vi è, infatti, un consistente corpus di ricerche basato sui geo-social media, fra cui i tweet geolocalizzati (De Falco et alii 2021). Si evidenziano quattro approcci esistenti a questi dati: il primo usa i dati provenienti dai geo-social media per sviluppare metodologie e tecniche finalizzate all'individuazione e alla gestione delle situazioni di emergenza (Nurwidyantoro e Winarko 2013). Il secondo approccio mira a individuare la distribuzione geografica di alcuni fenomeni sociali, come ad esempio la distribuzione dei gruppi etnici nelle grandi città (Longley 2015) o la diversità linguistica (Zhao e Cao 2017). Il terzo approccio, principalmente interessato da studi qualitativi, indaga se e in che modo ciò che accade nel modo online influenza le dinamiche nel mondo offline (Sutko e De Souza Silva 2010; Rzeszewski 2018). Infine, il quarto approccio, attraverso lavori principalmente quantitativi, tenta di spiegare la variabilità del fenomeno osservato online ricorrendo alle caratteristiche dello spazio offline. Ed è proprio quest'ultimo approccio ai geo-social media che caratterizza la ricerca qui presentata. L'assunto di fondo da cui parte il lavoro è che - nonostante sul digitale tutto risulti compresente - le opinioni, le percezioni e il sentiment relativi alla pandemia siano influenzati da due dimensioni di prossimità spaziale: la diffusione del contagio e la regolazione istituzionale. Dimensioni che assumono significato se inserite in una cornice spaziale ben precisa, che nel nostro caso coinciderà con il livello regionale. 
Al fine di contribuire alla letteratura sul tema COVID entro un frame di matrice ecologica (Zajczyk 1991) lo scopo di questo lavoro è focalizzare l'attenzione sull'analisi dei tweet durante la seconda ondata per individuare differenze in termini di opinioni, percezioni e sentiment fra le regioni in relazione sia al tipo di impatto del virus, ovvero la geografia del contagio, sia rispetto alle misure a cui sono state sottoposte seguendo la «geografia delle misure di contrasto al virus».

\section{LA COSTRUZIONE DEL DATO IN AMBIENTE DIGITALE: SCENARI NORMATIVI, CONTESTI TEMPORALI E MODALITA’ DI ANALISI SPAZIALE DEI TWEET}

La scelta degli strumenti di estrazione/organizzazione/costruzione e di analisi dei dati sono momenti molto delicati nell'approccio che fa capo ai metodi digitali (Rogers 2013) poiché da queste scelte dipende la capacità di elaborazione e di formulazione di una risposta all'obiettivo conoscitivo che dà vita alla ricerca presentata partendo dall'analisi non intrusiva su un particolare insieme di dati secondari riconoscibili come digital platform social data. L'estrazione dei tweet è avvenuta tramite tecniche automatizzate che fanno riferimento allo streaming API (Application Programming Interface) di Twitter, avviate in ambiente $R$ grazie alle funzioni del pacchetto «rtweet» che consente di dialogare con Twitter e di settare le procedure adeguate ai nostri standard di estrazione. Questi rispondono a: 1) limiti temporali-normativi, 2) chiavi testuali di ricerca e 3) caratteristiche del dato spaziale (tweet).

Relativamente al primo punto, la rilevazione è stata giornaliera, in un arco di tempo che ha coperto quasi tre mesi, dal 24 ottobre al 18 dicembre 2020, attraversando tre momenti scanditi da provvedimenti normativi nazionali volti al contrasto della diffusione del virus da COVID-19. Il primo, qui rinominato «limitazioni generali», riguarda le disposizioni normative generali del DPCM del 13 ottobre 2020 che, in seguito ad una prima recrudescenza del virus, reintroducono alcune restrizioni generali tra cui l'uso obbligatorio della mascherina all'aperto e al chiuso, il rispetto delle misure di sicurezza anche nelle abitazioni private in presenza di persone non conviventi, e la raccomandazione di evitare feste; il secondo momento normativo, qui definito «semafori», è relativo all'introduzione degli scenari di rischio del DPCM del 3 novembre 2020, che per la prima volta dall'inizio della pandemia, definisce una differenziazione delle misure per le regioni, a seconda della gravità della situazione epidemiologica, stabilita attraverso un set di indicatori sanitari. Tale differenziazione è basata su scenari cromatici di rischio: giallo, arancione, rosso, i quali prevedono normative sempre più stringenti rispetto alla tonalità della gradazione conferita allo scenario. Questa classificazione di rischio sarà ripresa, in chiave analitica e comparativa, per definire la «geografia delle misure di contrasto». Il terzo ed ultimo momento normativo, qui ridefinito come «disposizioni per Natale», si rifà al DPCM del 4 dicembre 2020 il quale conferma le disposizioni precedenti sulle fasce di rischio e introduce alcune limitazioni per le imminenti feste natalizie. Si vieta ogni spostamento in entrata o uscita dalle regioni, a qualsiasi scenario esse appartengano, a partire dal 21 dicembre 2020 e fino al 6 gennaio 2021, e ogni spostamento al di fuori del proprio comune di residenza il 25 e il 26 dicembre 2020, e il primo gennaio 2021.

Il secondo fattore che ha contribuito alla costruzione del dataset è relativo alle chiavi testuali di estrazione dei tweet, corrispondenti agli hashtag relativi ai topic trend nel giorno di avvio dell'estrazione (24 ottobre). Questi sono stati raggruppati secondo tre connotazioni tematiche: generica, normativa ed emotiva, ed altrettanti punti temporali di aggiornamento, in cui sono state introdotte nuove chiavi di ricerca in seguito alla normale evoluzione dei topic sul tema (ad esempio gli hashtag sul colore delle zone a novembre, o gli hashtag sui vaccini ad inizio dicembre) nel pieno rispetto dei tre motti dell'approccio basato sui metodi digitali, follow the medium, follow the native e follow the thing. La tabella 1 illustra gli hashtag usati per ciascuna fase e gruppo tematico.

Il terzo fattore di estrazione riguarda le caratteristiche del dato spaziale. Lo streaming API è stato impostato in modo da estrarre i tweet geolocalizzati per ricondurli alla regione da cui sono twittati. Sono stati esclusi i retweet, visto che la loro ripetitività testuale avrebbe fatto da "rumore" per la analisi del contenuto condotta sui testi. Il dataset così costituito è formato da 11.736 tweet. La tabella 2 mostra la distribuzione regionale dei tweet. Il periodo con più tweet, nel complesso, è la fase dei «Semafori» (3 novembre -4 dicembre). Tale valore non ci sorprende, considerato che è anche il periodo più lungo in termini di giorni. È tuttavia interessante se guardiamo la distribuzione regionale e i 
Tabella 1. Punti temporali di aggiornamento e connotazione tematica delle chiavi testuali di estrazione.

\begin{tabular}{|c|c|c|c|}
\hline Punti temporali & Generica & Normativa & Emotiva \\
\hline 24 ottobre & $\begin{array}{c}\text { \#Covid19 } \\
\text { \#Covid_19 } \\
\text { \#COVID-19 \#coronavirus } \\
\text { \#Covid19italia }\end{array}$ & $\begin{array}{c}\text { \#lockdown } \\
\text { \#dpcm } \\
\text { \#Lockdown2 \#secondaondata } \\
\text { \#lockdowncampania } \\
\text { \#coprifuoco }\end{array}$ & \#dittaturasanitaria \#nolockdown \\
\hline 23 novembre & & $\begin{array}{c}\text { \#zonagialla \#zonaarancione } \\
\text { \#zonarossa }\end{array}$ & \\
\hline 01 dicembre & $\begin{array}{l}\text { \#vaccini } \\
\text { \#vaccino }\end{array}$ & & \#vaccinoAntiCovid \\
\hline
\end{tabular}

Tabella 2. \% di tweet geolocalizzati per Regione Italiana nei tre periodi considerati.

\begin{tabular}{|c|c|c|c|c|}
\hline Regioni & Limitazioni Generali & Semafori & Disp.Natalizie & tot v.a. $(100 \%)$ \\
\hline$\overline{\text { Abruzzo }}$ & $18,7 \%$ & $55,3 \%$ & $26,0 \%$ & 219 \\
\hline Basilicata & $22,1 \%$ & $67,6 \%$ & $10,3 \%$ & 68 \\
\hline Calabria & $17,0 \%$ & $69,0 \%$ & $14 \%$ & 229 \\
\hline Campania & $28,1 \%$ & $53,4 \%$ & $18,5 \%$ & 809 \\
\hline Emilia-Romagna & $26,6 \%$ & $55,3 \%$ & $18,1 \%$ & 646 \\
\hline Friuli-Venezia Giulia & $27,6 \%$ & $52,0 \%$ & $20,4 \%$ & 196 \\
\hline Lazio & $29,4 \%$ & $48 \%$ & $22,6 \%$ & 2266 \\
\hline Liguria & $17,4 \%$ & $58,3 \%$ & $24,4 \%$ & 484 \\
\hline Lombardia & $26,8 \%$ & $54,8 \%$ & $18,4 \%$ & 3017 \\
\hline Marche & $23,9 \%$ & $52,1 \%$ & $23,9 \%$ & 234 \\
\hline Molise & $26,7 \%$ & $46,7 \%$ & $26,7 \%$ & 30 \\
\hline Piemonte & $24,3 \%$ & $57,2 \%$ & $18,5 \%$ & 736 \\
\hline Puglia & $22,5 \%$ & $59,6 \%$ & $17,9 \%$ & 386 \\
\hline Sardegna & $23,3 \%$ & $53,5 \%$ & $23,3 \%$ & 159 \\
\hline Sicilia & $23,7 \%$ & $54,5 \%$ & $21,8 \%$ & 705 \\
\hline Toscana & $18,7 \%$ & $57,4 \%$ & $24,0 \%$ & 718 \\
\hline Trentino-Alto Adige & $27,3 \%$ & $53,2 \%$ & $19,5 \%$ & 77 \\
\hline Umbria & $26,2 \%$ & $44,3 \%$ & $29,5 \%$ & 122 \\
\hline Valle d'Aosta & $18,8 \%$ & $61,3 \%$ & $20 \%$ & 80 \\
\hline Veneto & $33,5 \%$ & $46,5 \%$ & $20 \%$ & 555 \\
\hline tot $\%$ & $25,8 \%$ & $53,7 \%$ & $20,4 \%$ & 11736 \\
\hline
\end{tabular}

discostamenti significativi dal valore complessivo per ciascun periodo. Si osserva come per il primo periodo il Veneto sia la regione con più tweet, nel secondo invece Basilicata, Calabria e Valle d'Aosta (probabilmente per le polemiche legate all'attribuzione del colore per queste zone), e per il terzo periodo, Abruzzo e Umbria (si ricordano infatti le polemiche relative alla situazione abruzzese, che vede la regione in zona rossa per un periodo piuttosto prolungato).

La costruzione di quella che abbiamo definito la «geografia del contagio», intesa come diffusione territoriale del virus sull'intera Penisola, ha richiesto la creazione di una variabile sull'impatto del contagio per ciascuna regione. Si tratta del rapporto tra il numero di contagi sulla popolazione residente, categorizzato su tre livelli (basso, medio, alto). La tabella 3 offre una sintesi per ciascun periodo sulla quale sono state costruite le mappe che verranno presentate nel paragrafo che segue.

Infine, è riportato nel dataset il contenuto testuale dei tweet per cui si è fatto ricorso all'uso di tecniche lessicometriche del dato testuale e applicazione di tecniche automatiche di topic modeling e clusterizzazione in base ai tre momenti normativi considerati tramite software T-lab al fine di riscostruire la «geografia delle narrazioni» preva- 
Tabella 3. Impatto dei contagi per regione e per periodo normativo.

\begin{tabular}{lccc}
\hline Regioni & Limitazioni generali & Semafori & Disp.natalizie \\
\hline Abruzzo & medio & medio & medio \\
Basilicata & basso & basso & basso \\
Calabria & basso & basso & basso \\
Campania & medio & alto & medio \\
Emilia-Romagna & medio & medio & alto \\
Friuli VG & medio & alto & alto \\
Lazio & medio & medio & medio \\
Liguria & alto & medio & basso \\
Lombardia & alto & alto & medio \\
Marche & basso & basso & medio \\
Molise & basso & basso & medio \\
Piemonte & alto & alto & alto \\
Puglia & basso & basso & alto \\
Sardegna & basso & basso & basso \\
Sicilia & basso & basso & medio \\
Toscana & alto & medio & basso \\
Trentino AA & alto & alto & alto \\
Umbria & alto & medio & alto \\
Valle d'Aosta & alto & alto & basso \\
Veneto & medio & & basso \\
\hline
\end{tabular}

lenti in ciascuna regione per ciascun periodo individuato e stabilire le relazioni tra la dimensione spaziale, quella normativa e quella diffusiva del contagio come possibili elementi di impatto sulla costruzione delle narrative sul COVID-19 nella Twitter-sfera italiana.

\section{GEOGRAFIA DEL CONTAGIO, GEOGRAFIA DELLE MISURE DI CONTRASTO E GEOGRAFIA DELLE NARRAZIONI}

Come anticipato, nel paragrafo che segue si proveranno a connettere le diverse componenti che impattano sulla costruzione del racconto social attorno al COVID-19 che possono essere desunte dalla geografia di alcune distribuzioni regionali identificabili come: COVID-Spread, ovvero geografia della diffusione del contagio differenziale sulla Penisola; COVID-Measures, intesa come geografia delle misure di contenimento sviluppate in maniera differenziale a seguito dei provvedimenti calati sul territorio; COVID-Issues, identificabile come geografia delle narrazioni emergente dalla Twitter-sfera italiana. Per capire se ci siano correlazioni tra queste tre geografie diffusive di seguito si discuterà della loro particolare caratterizzazione in tre punti temporali precisi che corrispondono a tre momenti normativi decisivi nell' indirizzo delle narrazioni sull'epidemia da COVID-19 precedentemente descritti e definiti come «limitazioni generali», «semafori», e «disposizioni per Natale». Prima di entrare nello specifico delle narrazioni rintracciate però, è stato opportuno sintetizzare il contenuto tematico dell'intero corpus di tweet analizzato sviluppando un'analisi automatica che consentisse l'estrazione dei topic più rilevanti nel corpus. A tal proposito è stata applicata alla base dati una procedura probabilistica di topic modeling di tipo bottom-up che ci ha consentito di estrarre 20 temi rilevanti diversamente connotati attraverso i quali è possibile classificare i tweet analizzati conferendogli una precisa dinamica narrativa. I 20 temi estratti possono essere a loro volta organizzati in quattro macrodimensioni riportati in figura 2 e così definibili:

- Dimensione comunitaria del racconto (cerchi in rosso in alto a destra): cronaca del lockdown; sanità, diritti, lotta

e spesa pubblica; normativa e comportamenti protettivi da adottare; pandemia e senso di comunità; chiusure del sistema scolastico e di attività economiche; 


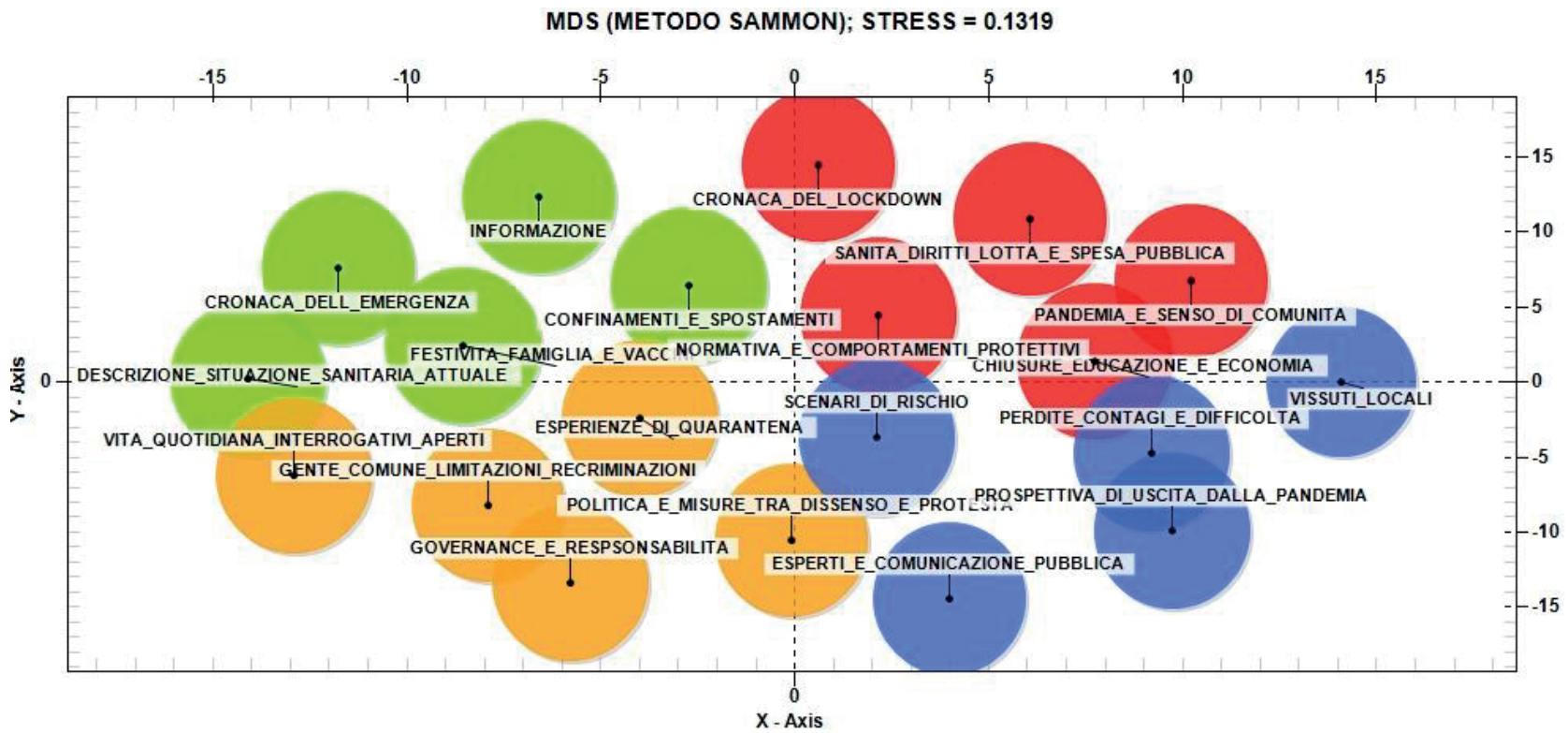

Figura 2. Mappa multidimensionale dei temi suddivisi per dimensione di prevalenza. Fonte: elaborazione su T-Lab corpus di 11.733 tweet e 1.823 lemmi, procedura di topic modeling probabilistica bottom-up su intero corpus e selezione di 20 aree tematiche. Modalità di rappresentazione con multidimensional scaling.

- Dimensione tecno-scientifica ed amministrativa del racconto (cerchi in blu in basso a destra): vissuti locali con centralità territoriale; perdite contagi e difficoltà esperite; prospettiva di uscita dalla pandemia; scenari di rischio e comunicazione istituzionale; esperti e comunicazione pubblica;

- Dimensione informativa del racconto (cerchi in verde in alto a sinistra): informazione; confinamenti e spostamenti; cronaca dell'emergenza; festività, dinamiche familiari e arrivo dei vaccini; descrizione della situazione sanitaria attuale;

- Dimensione governativa del racconto (cerchi in giallo in basso a sinistra): esperienze di quarantena; vita quotidiana interrogativi aperti; gente comune, limitazioni e recriminazioni; governance e responsabilità di istituzioni e politica; politica e misure tra dissenso e protesta.

Ciascuna delle macro-dimensioni individuate e dei temi che la compongono recupera diverse polarità del discorso che vanno da speranze per il futuro alle recriminazioni e alla sofferenza individuale e collettiva. Per comprendere come queste polarità si leghino al contesto di diffusione del virus e di emanazione di misure di contenimento, dunque ai loro impatti sulla delimitazione di percezioni e narrazioni, l'analisi per singoli periodi ci consente di clusterizzare in gruppi omogenei queste narrative emergenti definendole per prevalenza rispetto ai territori che le producono. Di seguito sono, pertanto, discusse in maniera separata le diverse connotazioni per periodo, mentre si ricostruiranno comparazioni e concordanza nel paragrafo a seguire.

\subsection{Covid-spread, Covid-measures e Covid-issues nel periodo «limitazioni generali»}

Stando a quanto riportato in figura 3, nel periodo definito di «limitazioni generali» la situazione di diffusione del contagio sulla Penisola, rappresentata a sinistra con la mappa degli impatti del contagio sulla popolazione, vede un andamento piuttosto generalizzato di massiccia diffusione su tutte le regioni a nord e centro inclusa la Campania (impatti tra medio e alto) e fatta eccezione per le Marche. Il Sud e le Isole rappresentano ancora scenari a basso impatto sulla popolazione, tuttavia per carenze generalizzate a livello di prevenzione, assistenza, diagnostica e strutture sanitarie capaci di fronteggiare le necessità imposte dall'emergenza, se si passa dalla mappa del contagio alla 

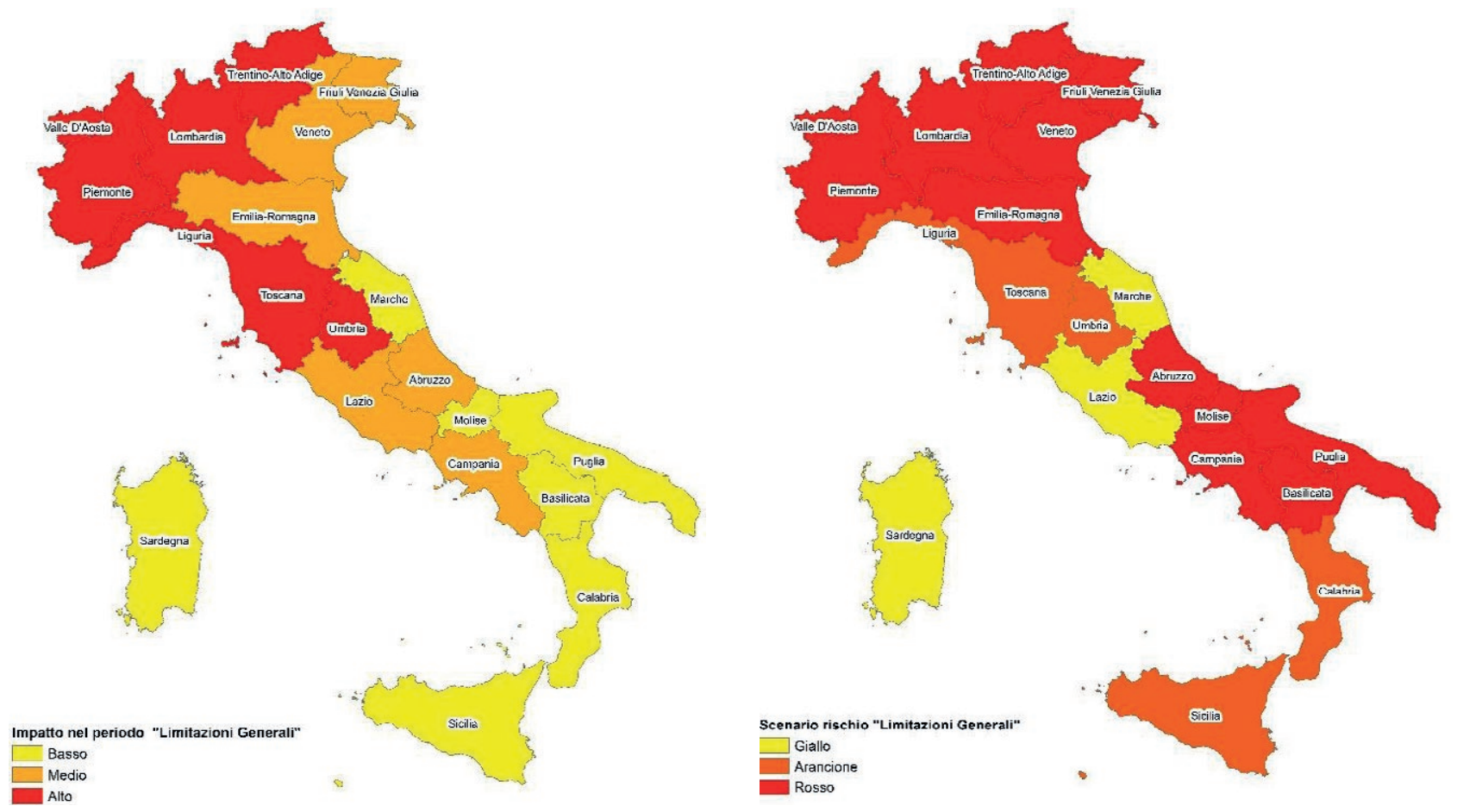

Figura 3. Sulla sinistra la mappa della «geografia del contagio» e sulla destra la mappa della «geografia delle misure di contrasto» per il periodo «limitazioni generali». Elaborazioni con $A R C$-GIS.

mappa delle zone costruita per scenari di rischio e riportata a destra della figura, scenari di allerta si registrano su tutta la Penisola ancora una volta con l'eccezione delle Marche seguite da Lazio e Sardegna. Un contesto di allerta generalizzato emerge da queste due rappresentazioni ampiamente dibattute nella loro portata sui media sia mainstream sia social tanto da far presumere un'influenza di questi due scenari diffusivi sulle percezioni e le narrazioni che emergono nei contenuti social user generated lasciati dagli utenti su Twitter.

Analizzando i tweet estratti per questo periodo, che corrispondono al 26,82\% del corpus di tweet considerato, sei gruppi di narrazioni possono essere distinti e disposti lungo un continuum per polarità di sentimento recuperate. Da sottolineare che ben tre su sei gruppi si connotano per un sentimento negativo che va dalla recriminazione, alla critica, alla sottolineatura di una sofferenza che sta dietro le limitazioni imposte. Il gruppo che recupera maggiore positività è quello concentrato sulla discussione di interventi di governance, spirito di comunità emergente e racconti di vita quotidiana. A contenuto nutro e per lo più informativo possono essere annoverati i gruppi relativi alla discussione della dimensione sanitaria sia dal punto di vista della gestione istituzionale sia dal punto di vista dei cittadini. A carattere decisamente negativo-critico possono essere evidenziati i gruppi basati su recriminazioni rispetto a normativa ed expertise e alle chiusure e le misure di confinamento dal punto di vista della dimensione economica da una parte e dal punto di vista della dimensione sociale, dei vissuti di quarantena e della prospettiva delle festività dall'altra. In figura 4 se ne propone la distribuzione ponendo in evidenza i gruppi colorati in base alla polarità (verde per il polo positivo, giallo per quello neutro e rosso per quello negativo) proiettando nello spazio fattoriale riprodotto i 20 temi estratti attraverso la procedura di modellizzazione automatica e le indicazioni rispetto agli scenari di impatto e di rischio. Già guardando a questa prima proiezione si può notare una vicinanza tra il racconto che emerge tra zone dichiarate a alto impatto o rosse e quelle a basso impatto o gialle, il che lascia presupporre che, al di là della diffusione del contagio e delle misure di contenimento con le loro particolari geografie, il discorso social della Twitter-sfera tende ad essere meno legato alla componente diffusiva territoriale mostrando una tendenziale carica negativa, critica e recriminatoria, in un periodo e in un momento vissuto in maniera generalizzata all'ombra di paure, tensioni e assenze di certezze per il prossimo futuro. 


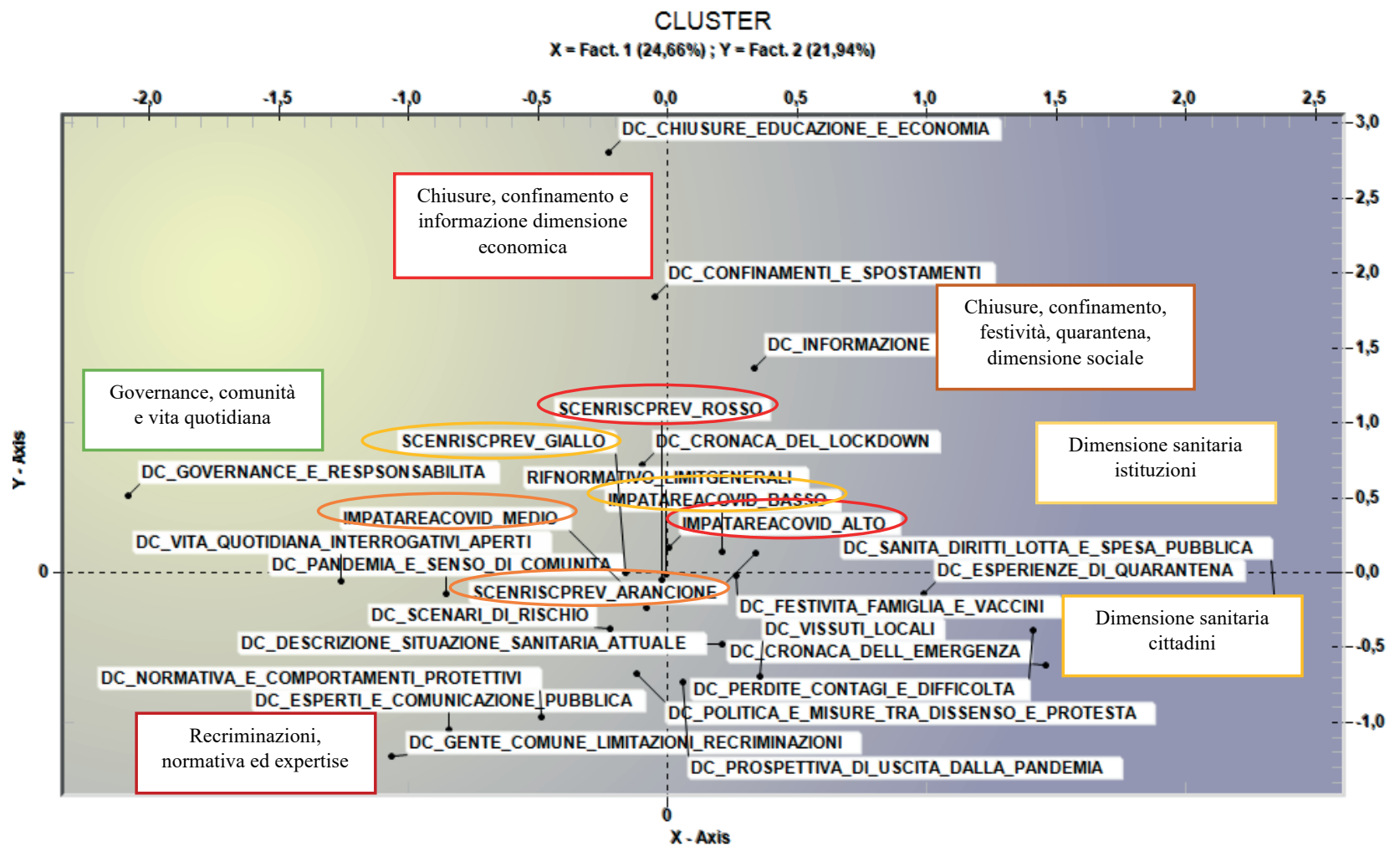

Figura 4. Cluster sui tweet del periodo «limitazioni generali» in base al contenuto tematico con proiezione in supplementare di scenari di impatto e scenari di rischio. Elaborazione con T-Lab.

La distribuzione di ciascun gruppo di narrazioni per regione può essere riprodotta come nel diagramma a composizione proposto in figura 5. Tuttavia, per comprendere l'articolazione delle narrazioni, è stato attribuito per prevalenza un solo gruppo di narrazione a ciascuna regione ottenendo, così, la «geografia delle narrazioni» riproposta in figura 6. Guardando a questa distribuzione, la correlazione tra alti impatti o zona rossa non è riscontrabile in tutte le regioni. Se questa è evidente nel Nord-Ovest del paese, lo è meno nel Nord-Est e nel Centro, che assieme al Sud e alle Isole, fatta eccezione per la Campania e l'Abruzzo, regioni tra le più colpite nella seconda ondata indagata in questo studio, dove le narrazioni emergenti vengono a connotarsi di maggiore neutralità e positività, con contenuti per lo più informativi e a carattere di racconto intimo o collettivo di stampo più marcatamente emotivo.

\subsection{Covid-spread, Covid-measures e Covid-issues nel periodo «semafori»}

Spostandoci sul secondo periodo normativo indagato, «semafori», in questo l'impatto della diffusione del contagio si ridimensiona in alcune zone del Nord, restando comunque particolarmente rilevante, e cresce anche nel Sud del Paese e questo andamento diffusivo è pienamente rispecchiato da una mappa degli scenari di rischio che resta colorata per prevalenza di zone rosse e arancioni in cui forti sono le limitazioni e crescenti le ansie per il fronteggiamento di una situazione sanitaria che diventa sempre più fuori controllo. Questo vedendo l'eccezione della Regione Veneto e ancora una volta di Lazio e Sardegna con aggiunta del Molise nelle quali la situazione viene tenuta maggiormente sotto controllo. Che effetto avrà il peggioramento dello scenario diffusivo sulle narrative?

In questo periodo i tweet recuperati crescono di volume tanto da rappresentare quasi la metà dell'intero corpus analizzato (il $52,51 \%$ per l'esattezza). I gruppi che vengono a distinguersi sono sempre sei, ma questa volta bilanciati 


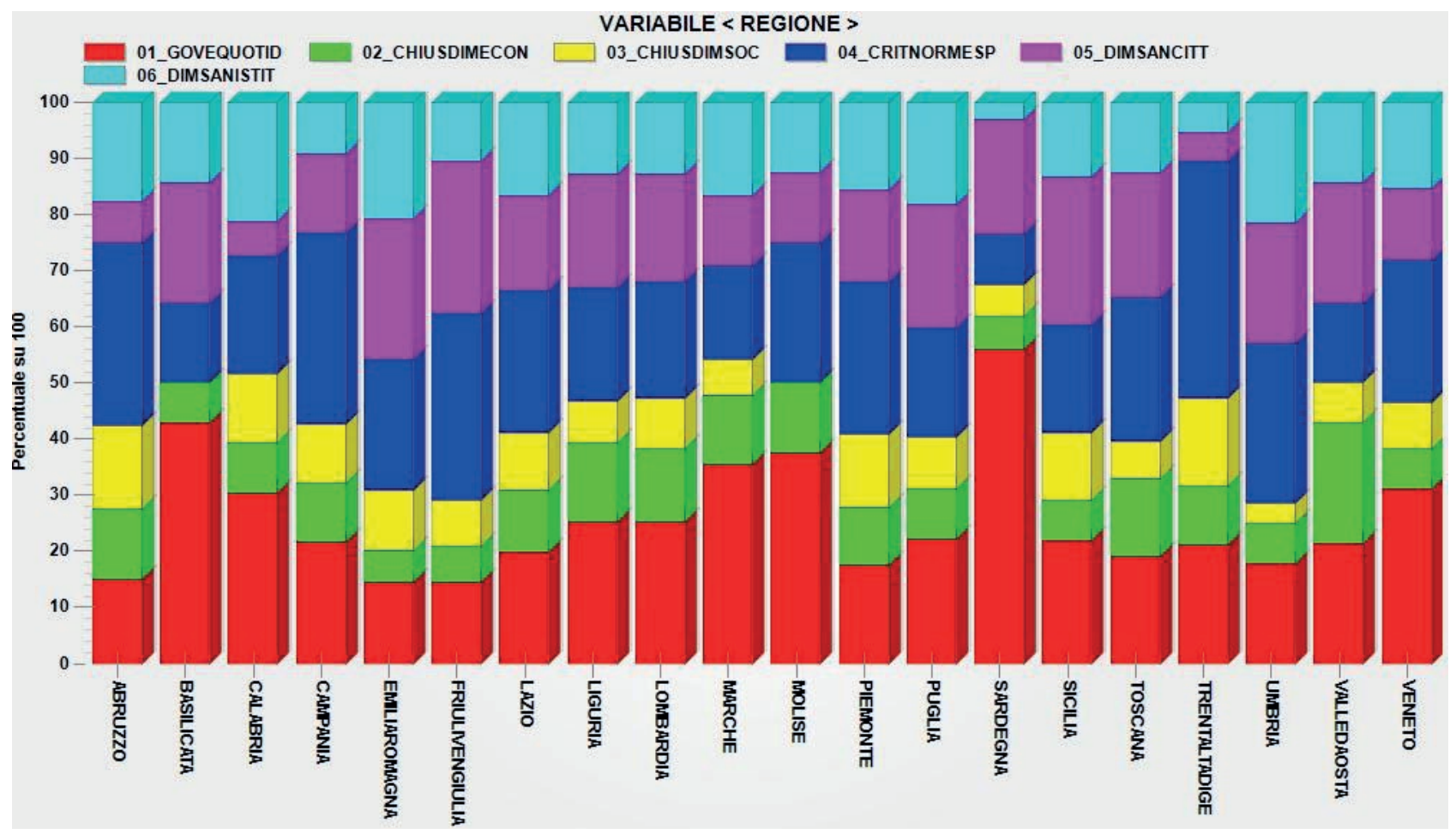

Figura 5. Diagramma a composizione della distribuzione dei gruppi di narrazioni emersi per ciascuna Regione nel periodo «limitazioni generali». Elaborazioni con T-Lab.

a due a due lungo il continuum di polarità. Per cui ritroviamo due classi positive definite come resilienza, responsabilità e senso di comunità, e discorsi sulla quotidianità e il futuro. Le due classi neutrali a contenuto informativo si concentrano su cronaca e informazione legata agli aggiornamenti sulla diffusione del virus e alle limitazioni imposte in base ai colori delle zone e relative alla governance dell'emergenza. Le due classi a contenuto negativo vertono invece sulla critica alle misure di confinamento e degli spostamenti così come sulle recriminazioni relative alla gestione politica ed amministrativa dell'emergenza. Guardando al piano fattoriale che propone gruppi di narrative, temi e senari di impatto e rischio, ancora una volta non c'è una netta separazione tra narrative negative e scenari ad alto impatto e, viceversa, tra narrative positive e scenari a basso impatto ancora una volta sottolineando una mancanza di connessione immediata tra dati di diffusione geografico-territoriale e narrazioni emergenti sul COVID-19.

E questo è ancora più evidente scendendo nel dettaglio della distribuzione dei gruppi di narrative per regione (figura 9) e nella loro riproduzione per prevalenza sulla Penisola (figura 10).

Infatti, diminuisce la rilevanza generale delle categorie negative e del loro peso nella distribuzione generale (si noti l'esiguità delle categorie colorate in blu e celeste nella figura 9 che corrispondono alle categorie negative) e queste continuano a prevalere dove l'evoluzione della diffusione del virus si è mostrata senza tregua, come nelle regioni Lombardia, Piemonte, Emilia-Romagna e Campania. Polarità tendenzialmente positive connotano regioni quali Valle d'Aosta, Abruzzo, Marche, Liguria e Sardegna nelle quali gli scenari di impatto e rischio sono fortemente diversificati. La connotazione neutrale a carattere informativo si espande a macchia d'olio nelle restanti regioni rimarcando anche la necessità di seguire con maggiore dovizia una normativa differenziale per scenari di rischi che cambia ogni 15 giorni e impone regole e misure differenti a seconda delle variazioni del momento. 


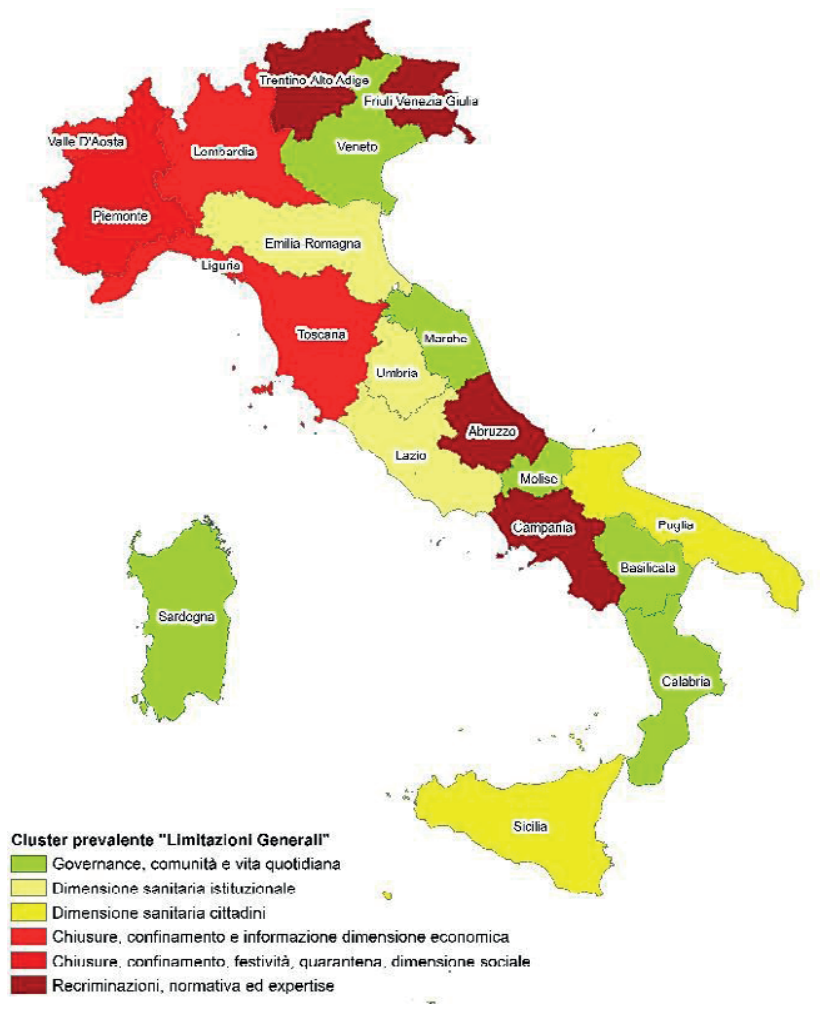

Figura 6. Mappa della «geografia delle narrazioni» per il periodo «limitazioni generali». Elaborazioni con ARC-GIS.

\subsection{Covid-spread, Covid-measures e Covid-issues nel periodo «disposizioni per Natale»}

Nel terzo periodo normativo considerato, «disposizioni per Natale», comincia ad essere evidente una tendenza al miglioramento della situazione epidemiologica in alcune regioni e il peggioramento in altre precedentemente non così colpite. Tuttavia, seguendo gli scenari di rischio, questi impatti sono attualmente gestibili e contenibili dalle regioni, tanto è vero che, guardando alla geografia delle misure di contenimento in figura 11 il rosso è mantenuto per una sola regione, l'Abruzzo, mentre tutte le altre transitano all'arancione e al giallo. La situazione muta enormemente rispetto al primo periodo considerato, in vista anche di una festività particolarmente importante a livello culturale ma soprattutto per la dimensione economica a questa connessa, il Natale. Gli allentamenti delle misure servono anche a preservare un'integrità sociale e una non letale chiusura delle attività commerciali che di questo periodo possono beneficiare a discapito di un anno di perdite, seppure non allentando completamente la stretta e mantenen-
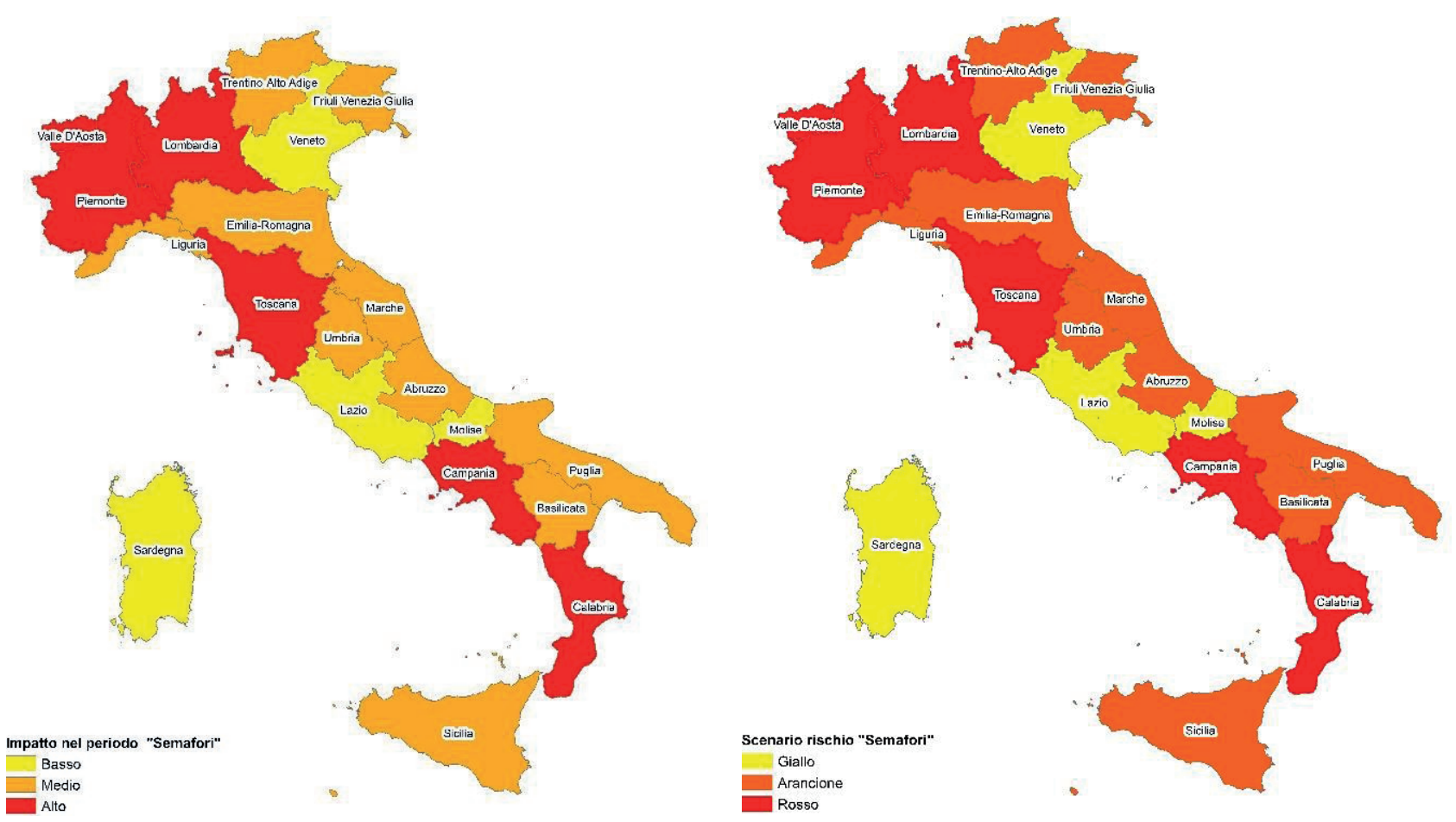

Figura 7. Sulla sinistra la mappa della «geografia del contagio» e sulla destra la mappa della «geografia delle misure di contrasto» per il periodo «semafori». Elaborazioni con $A R C$-GIS. 


\section{CLUSTER}

$X=$ Fact. $1(26,62 \%) ; Y=$ Fact. $2(22,9 \%)$

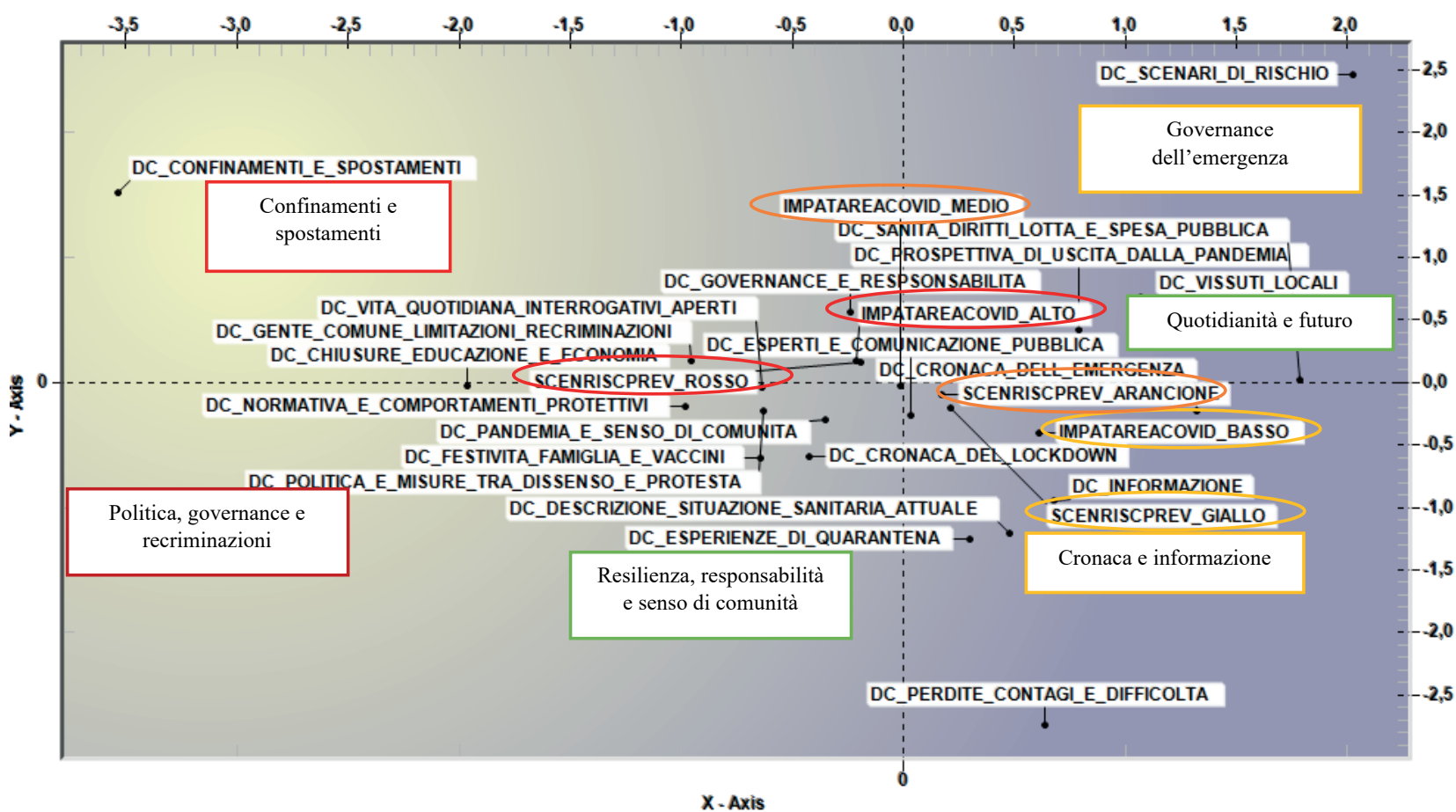

Figura 8. Cluster sui tweet del periodo «semafori» in base al contenuto tematico con proiezione in supplementare di scenari di impatto e scenari di rischio. Elaborazione con T-Lab.

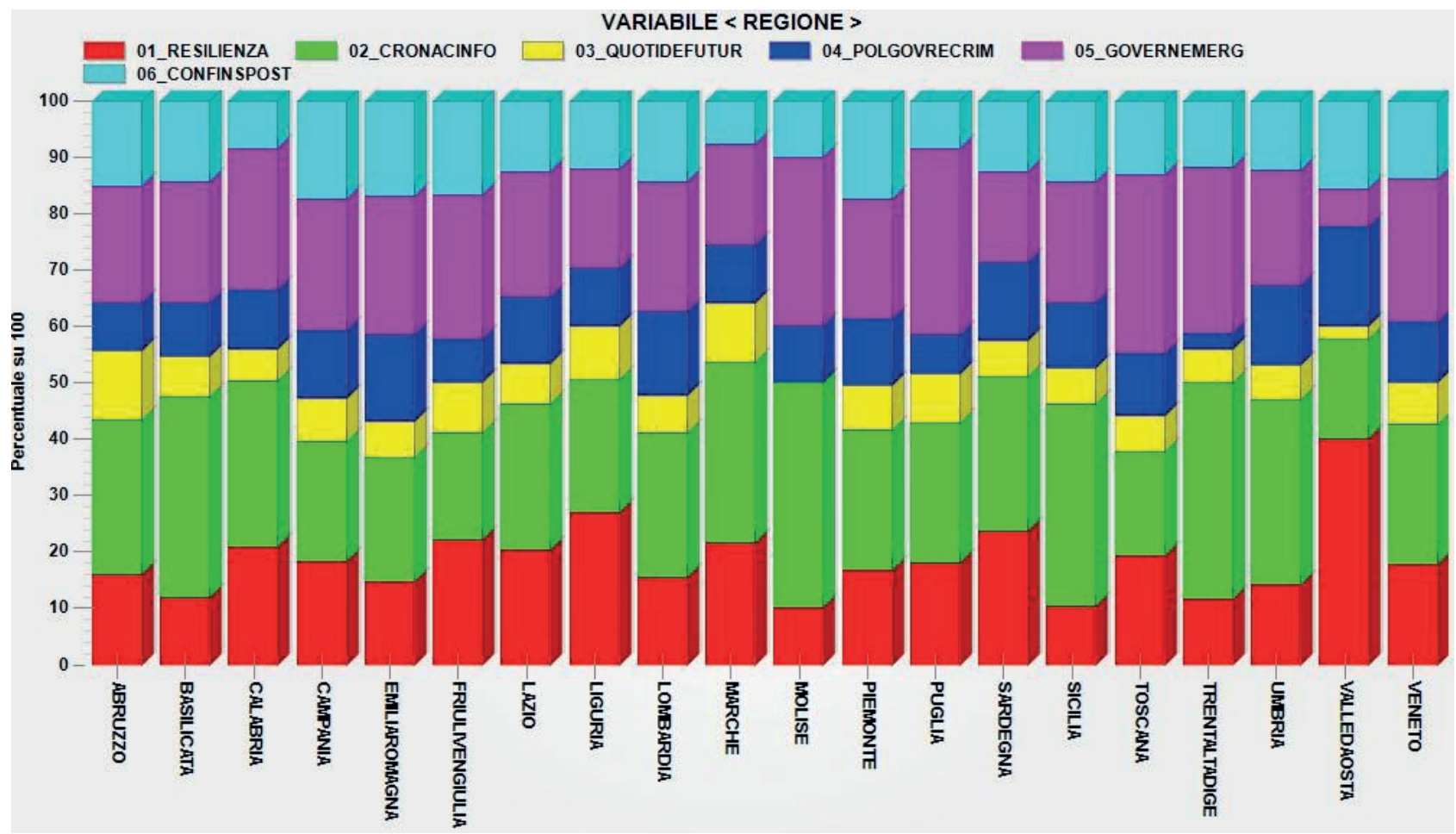

Figura 9. Diagramma a composizione della distribuzione dei gruppi di narrazioni emersi per ciascuna Regione nel periodo «semafori». Elaborazioni con T-Lab. 


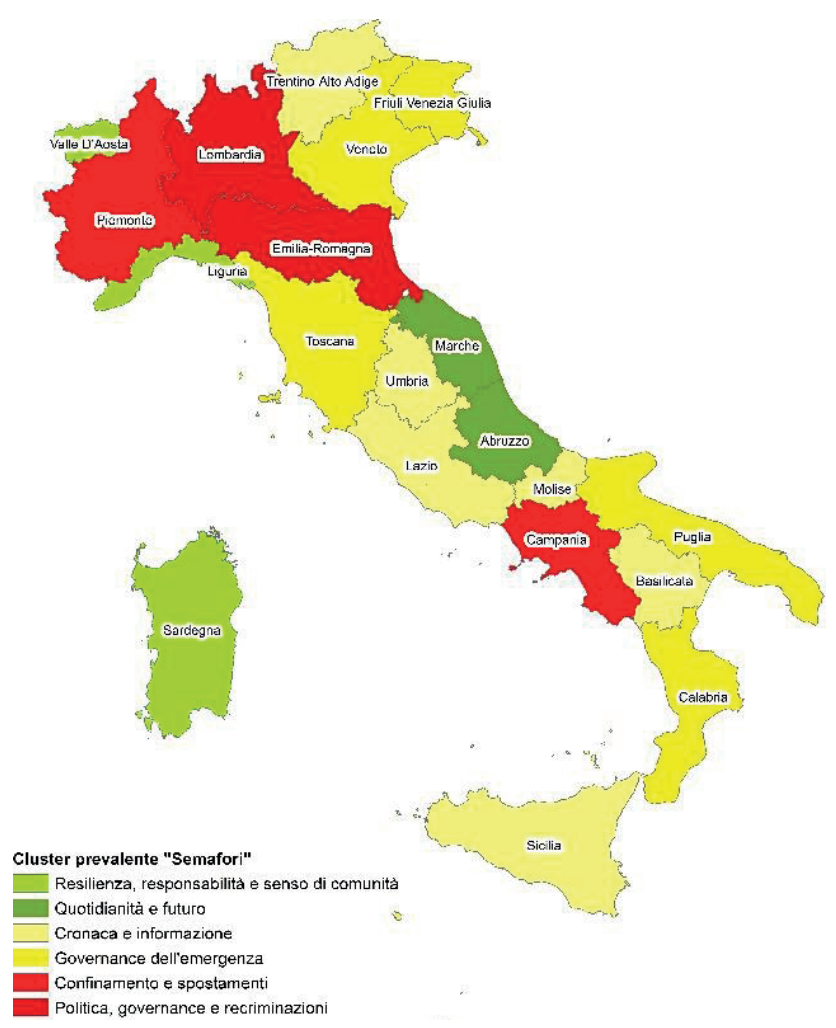

Figura 10. Mappa della «geografia delle narrazioni» per il periodo «semafori». Elaborazioni con ARC-GIS.

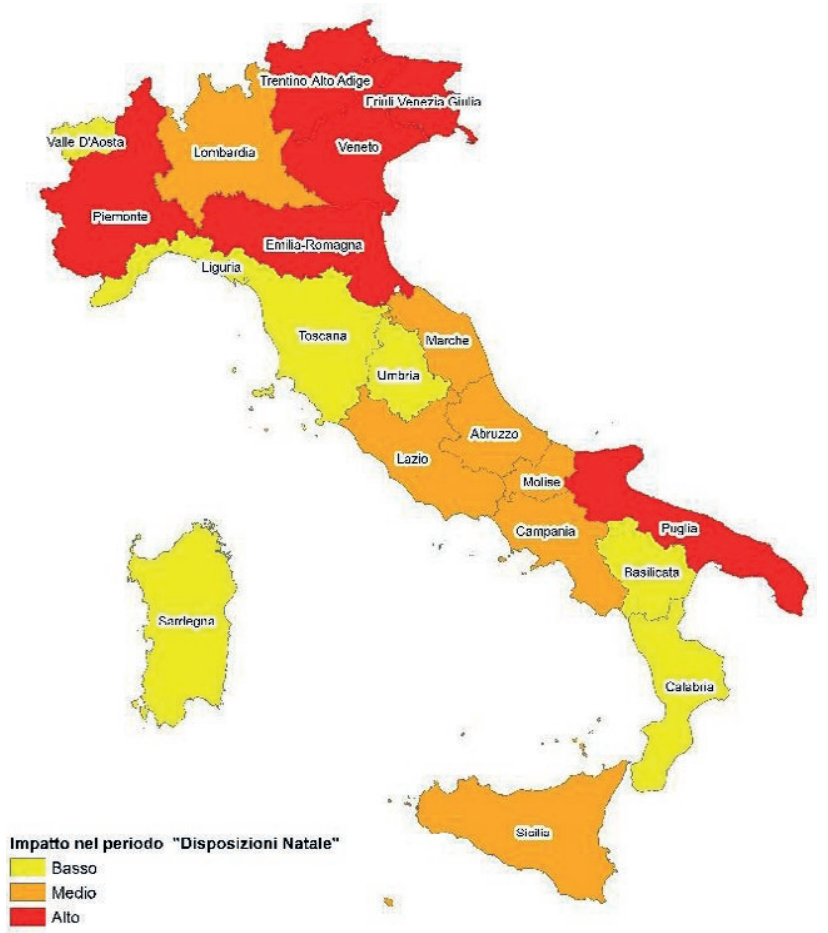

do alta la dimensione di allerta e il richiamo alla responsabilità dei singoli.

I tweet di questo periodo rappresentano il $20,66 \%$ del corpus indagato. I gruppi di narrative emergenti si riducono a 5 perdendo una categoria tra quelle positive. L'unica categoria positiva resta quella dedicata alla resilienza e al senso di comunità, seguita da due categorie neutrali concentrate sulla diffusione di informazioni su provvedimenti e scenari futuri e sulla più generale comunicazione dell'emergenza sanitaria. Le categorie critiche e negative si concentrano, invece, sulla narrazione dei vissuti individuali e della governance dell'emergenza sanitaria da una parte, e dall'altra su scenari di rischio, perdite e chiusure per il contenimento dall'altra. Anche nella rappresentazione fattoriale riprodotta in figura 12 gli scenari di impatto e di rischio non si distribuiscono separatamente tra i tipi di narrative emergenti e anche in questo periodo può essere presupposta una sorta di rapporto slegato tra andamenti diffusivi e narrazioni.

Se la situazione epidemiologica muta verso uno scenario maggiormente positivo, lo stesso non si può dire, però, delle narrative emergenti, ormai percorse

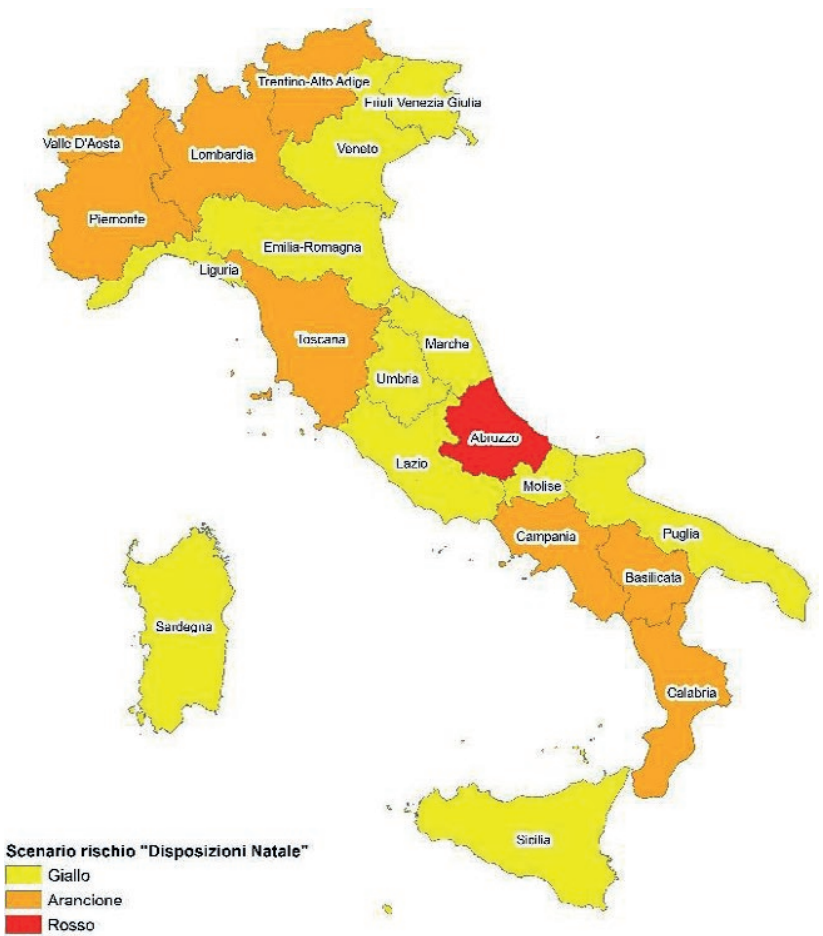

Figura 11. Sulla sinistra la mappa della «geografia del contagio» e sulla destra la mappa della «geografia delle misure di contrasto» per il periodo «disposizioni per Natale». Elaborazioni con ARC-GIS. 


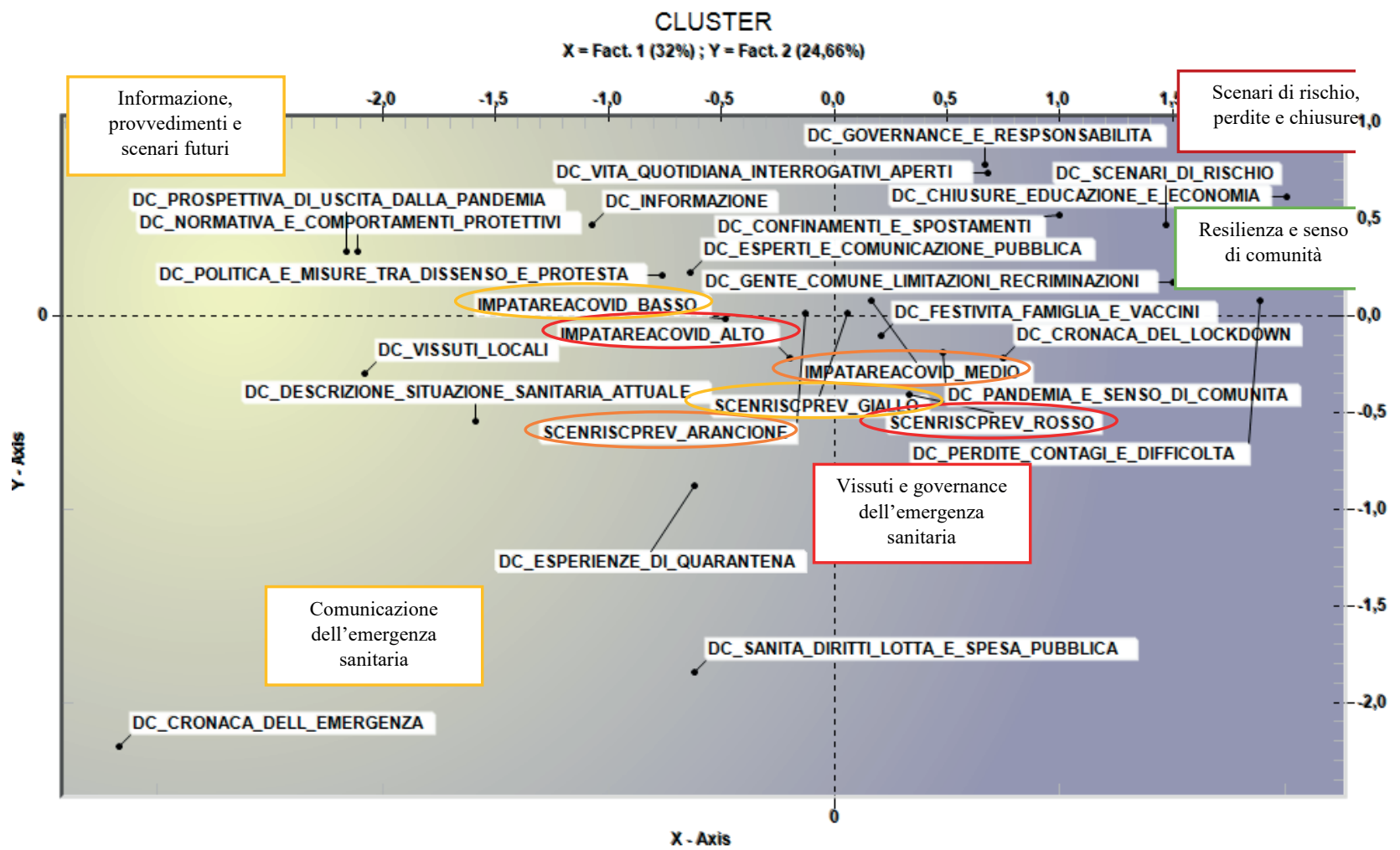

Figura 12. Cluster sui tweet del periodo «disposizioni per Natale» in base al contenuto tematico con proiezione in supplementare di scenari di impatto e scenari di rischio. Elaborazione con T-Lab.

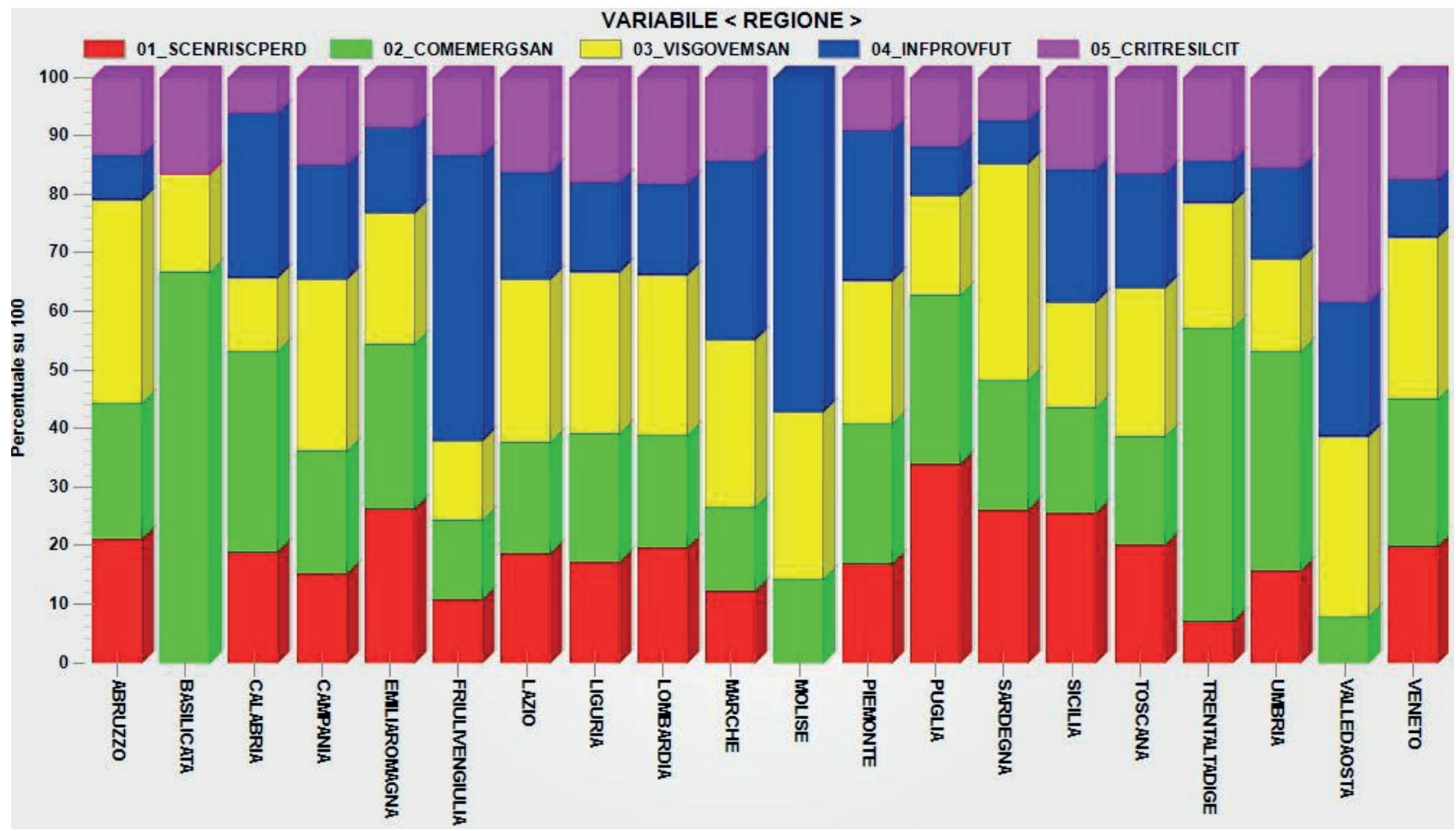

Figura 13. Diagramma a composizione della distribuzione dei gruppi di narrazioni emersi per ciascuna regione nel periodo «disposizioni per Natale». Elaborazioni con T-Lab. 


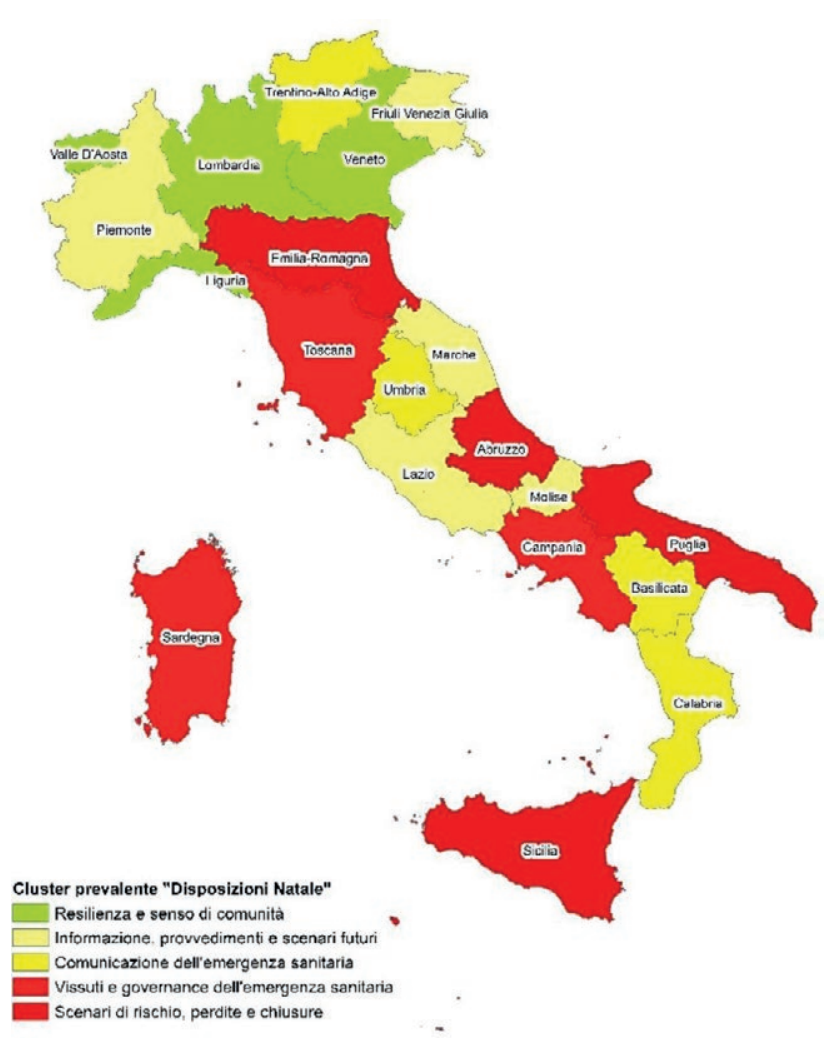

Figura 14. Mappa della «geografia delle narrazioni» per il periodo «disposizioni per Natale». Elaborazioni con ARC-GIS.

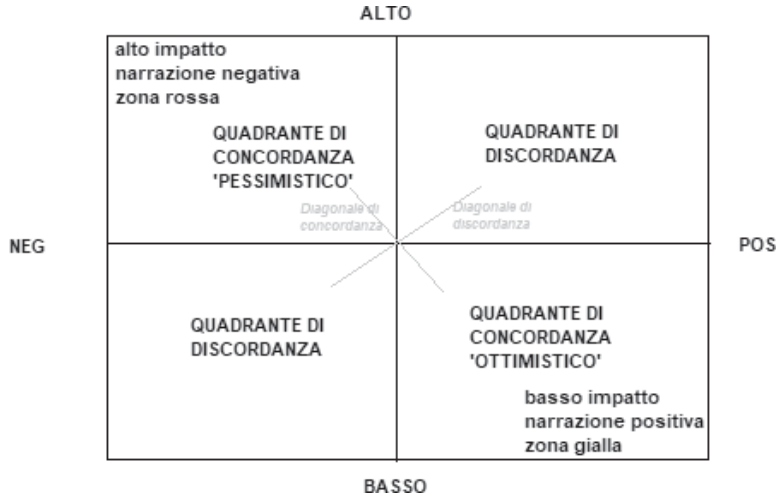

Figura 15. Quadro tipologico del piano di concordanza / discordanza tra le tre componenti.

da prolungate situazioni di restrizioni, rigore, perdite e sacrifici, per cui la Penisola torna a colorarsi di rosso nel Centro, al Sud e nelle Isole, se non per gli scenari di rischio e le misure connesse, proprio per la polarità delle narrazioni emergenti (figura 14). Le narrazioni positive si fanno residuali (si veda figura 13 classe colorata in viola), eppure risultano prevalere in regioni che hanno vissuto e vivono scenari epidemiologici particolarmente preoccupanti come Lombardia, Veneto, Valle d'Aosta e Liguria. Restano ancora di particolare rilievo le categorie neutrali a carattere informativo proprio perché continua ad essere di particolare importanza l'aggiornamento rispetto ai provvedimenti differenziali, in questo periodo non solo per aree geografiche ma anche per giorni festivi e prefestivi relativi al periodo Natalizio.

\section{IL PIANO DELLE CONCORDANZE E DELLE DISCORDANZE TRA TERRITORI. UN TENTATIVO DI SINTESI TRA NARRAZIONE, SITUAZIONE EPIDEMIOLOGICA E DISPOSIZIONI NORMATIVE}

Il carattere monotonico delle tre proprietà che abbiamo fin qui considerato, COVID-Issue, COVID-Spread e COVID-Measures ci incoraggia a tentare una sintesi per identificare i gradi di concordanza/discordanza fra i tre livelli, per ciascuna regione. In tal senso, la concordanza tra livelli rappresenta anche la situazione attesa. Ad esempio, una situazione di criticità dei contagi (alto impatto) dovrebbe, in linea teorica, prefigurare un contesto di restrizioni elevate (zona rossa) e, verosimilmente, una narrazione connotata da sentiment negativo. Al fine di rilevare visivamente e in forma simultanea gruppi di regioni con profili di concordanza/discordanza simili, abbiamo deciso di rappresentare un piano in cui l'asse delle ascisse delinea le COVID-Issue, l'ordinata è relativa al COVIDSpread, mentre le COVID-Measures sono rappresentate dal colore delle etichette delle regioni, scelta che garantisce la tridimensionalità del piano. Pertanto, è stato possibile tratteggiare due diagonali: una che abbiamo definito «diagonale della concordanza» che individua due quadranti di concordanza, uno «ottimistico», l'altro «pessimistico» in relazione, ovviamente ai tre fattori-COVID; l'altra diagonale individua, alla stessa maniera, i profili di discordanza. L'incrocio tra gli assi rappresenta il punto medio. La figura 15 illustra sinteticamente i possibili incroci di concordanza e discordanza. 


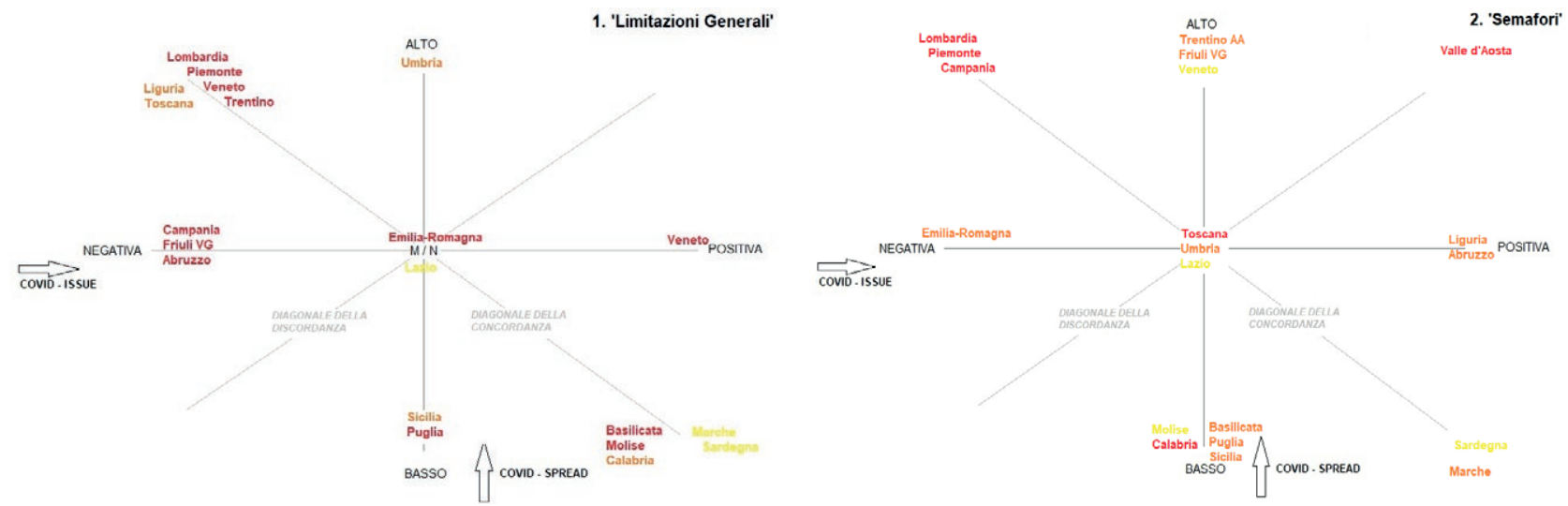

Figura 16. Piano di «Limitazioni Generali».

Figura 17. Piano del periodo «Semafori».

I punti «Regione» sono stati proiettati per ciascuno dei tre periodi considerati. Nel primo periodo, «limitazioni generali» (fig. 16), si osserva un "addensamento" maggiore sulla diagonale della concordanza, soprattutto sul "versante" pessimistico, con Lombardia, Piemonte, Veneto e Trentino-Alto Adige, quindi zone rosse con un impatto preoccupante dei contagi e un buzz social tendenzialmente negativo, che sono opposti a Marche e Sardegna, zone gialle, e situazione COVID, percepita ed effettiva, tutto sommato contenuta. Si trovano nel quadrante della concordanza, seppur con colori normativi non coerenti, anche le regioni Liguria e Toscana, nel quadrante «ottimistico», con Basilicata, Molise e Calabria nel quadrante «pessimistico». In questa prima fase una buona parte delle regioni presenta, quindi, una struttura narrativa piuttosto coerente con il contesto dell'emergenza. Questo momento preliminare della seconda ondata sembra non offrire spazio a situazioni contraddittorie, tant'è che la retta diagonale che attraversa i quadranti di discordanza non presenta alcun «punto-Regione».

Relativamente al periodo «semafori» (fig. 17), alcune delle Regioni presenti sulla diagonale della concordanza "emigrano" sull'asse delle ascisse o dell'ordinata, mentre una regione appare sulla diagonale della discordanza: è la Valle d'Aosta, che al netto di una situazione critica dal punto di vista epidemiologico e normativo (la Valle d'Aosta in questo periodo è prevalentemente zona rossa) sembrerebbe, stando ai "cinguettii" dei residenti, non accusare la gravità della situazione in corso. La seconda ondata sembrerebbe non influire più sul mood della Twitter-sfera: molte regioni infatti si trovano sull'ordinata, segno che dal punto di vista della narrazione non si registrano evidenti sbalzi di sentiment. Così non è, o almeno non ancora, per le condizioni epidemiologiche: in alcune regioni (soprattutto del Sud) il contagio rallenta, in altre (Nord-est) non sembra arrestarsi.

Nel terzo scenario relativo alle «disposizioni per Natale» (fig. 18) si verifica una distribuzione

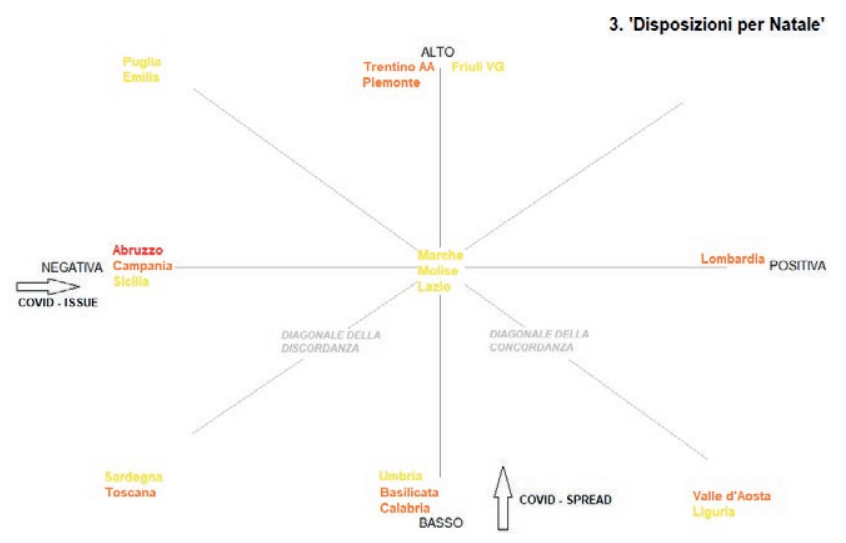

Figura 18. Piano del periodo «Disposizioni per Natale». più eterogenea delle regioni sul piano. Il ritorno di molte aree in zona gialla non sembra sortire effetti positivi sul sentiment della narrazione. Delle tre regioni che si trovano nel semi-quadrante della narrazione positiva, soltanto una, difatti, è in zona gialla. Interessante è il caso dell'Abruzzo, che al netto di una situazione epidemiologica apparentemente non critica, è l'unica regione che rimane in zona rossa. Non è banale l'ipotesi per cui questa situazione, a prima vista contraddittoria, sia in qualche modo in relazione con la comunicazione prevalentemente sfavorevole dei tweet provenienti da quell'area. 
Complessivamente, l'osservazione dei tre piani ci suggerisce che la seconda ondata dell'emergenza, almeno per quanto riguarda le tre componenti osservate, ha avuto un'evoluzione non lineare, descrivendo, in alcuni casi, anche situazioni piuttosto contraddittorie. Ad un primo momento non di difficile interpretazione, con gruppi consistenti di regione "rosse" a polarità narrativa positiva ed altre regioni "gialle" a polarità positiva, sono seguite altre due fasi in cui, progressivamente, la relazione tra contagi, narrazione e normativa è sembrata frammentarsi. Non solo, anche le regioni non hanno quasi mai mantenuto le proprie posizioni lungo questo nostro continuum temporale, segno di una situazione in rapida trasformazione. Come se, per ragioni che in qualche modo abbiamo ritrovato nell'analisi del contenuto precedente, il racconto dell'emergenza sia andato in altre direzioni rispetto a quanto il contesto normativo ed epidemiologico potessero indirizzare.

\section{DISCUSSIONI E CONCLUSIONI}

Nella prima fase della pandemia, attraverso diversi studi richiamati in introduzione e nel secondo paragrafo, è stato portato all'evidenza come la distribuzione territoriale delle narrazioni sul COVID-19 fosse connessa in modo inverso alla diffusione territoriale del contagio. In particolare, le regioni più colpite erano caratterizzate da narrazioni di tipo resiliente mentre quelle meno colpite manifestavano principalmente un sentiment negativo, espressione di una forte preoccupazione per la pandemia e le sue conseguenze. L'analisi dei tweet geolocalizzati, estratti durante la seconda fase della pandemia ed esplorati in questo studio, ha avuto lo scopo di controllare se il tipo di relazione emersa durante la prima ondata fosse cambiata o meno. L'analisi effettuata nella seconda fase ci ha restituito innanzitutto un quadro complesso e articolato, formato da molteplici prospettive, percezioni e punti di vista. Ed è proprio questo, infatti, uno dei punti di forza dell'analisi dei social media, ossia la possibilità di mappare, definire e descrivere tutti i significati e le associazioni attribuite ad un determinato topic oggetto di studio. L'analisi del contenuto dei tweet geolocalizzati ha messo in evidenza che nella seconda fase sono quattro le principali dimensioni narrative soggiacenti ai post. Oltre alla dimensione comunitaria del racconto, fatta di «cronache del lockdown», richiamo ai «comportamenti protettivi da adottare» e a questioni inerenti al vissuto quotidiano nel suo complesso, ad emergere sono state anche le dimensioni «governativa», «informativa» e «tecnico scientifica». Un risultato, questo, molto interessante che si connette e dà forza a quanto rilevato nei lavori precedentemente citati. Infatti, oltre che come mezzo per esprimere i propri stati d'animo, positivi o negativi, durante la pandemia i social sono stati utilizzati come mezzo per scambiarsi informazioni e discutere dei provvedimenti e delle misure adottate dai governi per il contenimento del virus. Gli stessi governi che, come visto, hanno lanciato campagne di comunicazione sui social su questioni riguardanti il COVID-19. Dentro questo quadro generale, l'analisi delle narrazioni prevalenti nei tre periodi individuati ha messo in luce innanzitutto la stanchezza di una popolazione che per la seconda volta, dopo una pausa estiva che lasciava ben sperare, ha dovuto affrontare nuove limitazioni congiuntamente alla ripresa della diffusione del virus. Ciò emerge osservando i cluster con sentiment negativo che sono stati individuati nei tre periodi. In particolare, nel primo periodo, quello delle «limitazioni generali», le narrazioni con sentiment negativo hanno riguardato principalmente le rinnovate chiusure decise dal governo, ed è da sottolineare come il primo periodo sia l'unico dei tre analizzati in cui il sentiment negativo si articola in tre cluster diversi dimostrando l'impatto più o meno traumatico che hanno avuto inizialmente le rinnovate limitazioni imposte dal Governo Conte. L'eco di questa iniziale frustrazione verso le decisioni governative si rileva anche nel secondo periodo, quello dei «semafori», poiché i due cluster con sentiment negativo racchiudono i tweet relativi alle misure di confinamento e alle scelte politiche che le hanno prodotte. Infine, nell'ultimo periodo analizzato, quello delle «disposizioni per Natale», il centro del sentiment negativo non è più rappresentato dalle limitazioni imposte alla libertà individuale in quanto il focus cambia e si sposta sugli effetti della pandemia sulla società e quindi sulla gestione dell'emergenza sanitaria e gli effetti di lungo termine che quest'ultima potrebbe avere. Nell'ultima fase, l'emergenza sanitaria e i suoi effetti diventano il core dei tweet con sentiment negativo mentre nelle fasi precedenti alcune questioni ad essa connesse (dimensione sanitaria; governance dell'emergenza, informazioni, ecc.) erano caratterizzate da un sentiment principalmente neutro. Nei tweet con sentiment positivo, infine, emerge abbastanza chiaramente come l'ele- 
mento soggiacente in tutti e tre periodi è rappresentato dal senso di appartenenza alla comunità. Il bene della comunità oltre che quello dei propri parenti e amici, rappresenta probabilmente l'unico frame entro cui dare una lettura positiva agli sforzi e i sacrifici imposti dalle misure di contenimento del virus. Qualora fosse stato possibile, sarebbe stato sicuramente interessante analizzare le caratteristiche socioeconomiche di ciascun gruppo, ma, come noto, uno dei principali limiti dell'analisi dei dati provenienti da Twitter, è dato dall'impossibilità di recuperare le informazioni socioeconomiche dei soggetti da cui provengono i tweet. Ciò che è invece possibile fare grazie all'utilizzo dei tweet geolocalizzati è analizzare l'influenza delle caratteristiche territoriali sul fenomeno analizzato, cosa che nel nostro è rappresentata dall'eventuale relazione, a livello regionale, fra la diffusione del virus e il tipo di narrazione prevalente. Ciò ci ha permesso di rispondere alla nostra domanda di ricerca e di rilevare quindi eventuali differenze nella spazialità delle narrazioni in relazione a quanto emerso nella prima fase della pandemia. Un primo risultato è dato dal fatto che, a differenza della prima fase, raramente si è osservata una discordanza fra narrazioni prevalenti e diffusione del virus. Nelle regioni dove le cifre giornaliere sul virus risultavano alte, o comunque preoccupanti, raramente sono state riscontrate narrazioni prevalentemente positive e viceversa. Per individuare il secondo risultato dell'analisi bisogna prendere in considerazione oltre alla diffusione del virus anche le misure differenziate che hanno interessato le regioni. Se da un lato emerge che lungo i tre periodi cala il numero di regioni in cui la relazione lineare fra diffusione del virus e tipo di narrazione prevalente esiste, dall'altro è possibile notare come nei tre periodi le regioni "rosse" raramente siano caratterizzate da una narrazione positiva. Ciò che emerge è che nella seconda ondata più che la diffusione territoriale del virus ad incidere sulle narrazioni regionali sono state i tipi di limitazioni imposte e quindi la fascia di rischio. Due casi, a nostro avviso esemplari, sono rappresentati dalla Lombardia e dalla Campania, entrambe interessate da livelli di diffusione del virus simile e da narrazioni prevalentemente negative nei primi due periodi. Nel terzo periodo, invece, tali narrazioni a parità di livello di diffusione del virus, diventano positive per la Lombardia (diventata zona arancione) e permangono invece negative in Campania, diventata arancione per il governo centrale, ma rossa invece per il governo regionale presieduto dal governatore De Luca, il quale favorevole a politiche più stringenti per contrastare la diffusione del virus, ha confermato le restrizioni e le limitazioni della fase precedente. In altre parole, quindi, oltre all'emergenza sanitaria ci sono state altri stressor come le limitazioni alle libertà personali che hanno inciso sul versante economico e sociale e che sono state fonte di percezioni negative. Tali percezioni avevano ragione di esistere laddove la fascia di rischio era alta con conseguente limitazione delle possibilità di spostamento e chiusura delle attività. Questa seconda fase di emergenza, dunque, è riconoscibile anche in questa «geografia» di paure, sentimenti e speranze: le abbiamo chiamate «voci digitali» alle quali abbiamo provato a dare sostanza attraverso la dimensione territoriale, quasi fosse un tentativo di renderle distinguibili in quell'enorme flusso comunicativo della social arena. Lo scenario che ne emerge invita a riflettere sulle conseguenze che può avere una trattazione non unitaria delle narrazioni social digitali fondata sulla dimensione fisico-spaziale della georeferenziazione delle stesse. Le misure di contenimento del contagio che adottano l'elemento spaziale di confinamento e di limitazione della circolazione provocano un'influenza tale nel discorso social da porsi come veri e propri elementi di rottura con funzione polarizzante (Sunstein 2007). Pur diventando oggetto del dibattito online, queste argomentazioni, nonché gli elementi di retorica argomentativa e di sentimento che veicolano, provocano influenze sulle narrazioni almeno quanto degli elementi di prossimità rappresentati dalla geografia di diffusione dei contagi differenziata nelle diverse regioni o di prossimità dell'esperienza che, a livello individuale, induce ad avere avuto un'esperienza diretta di contagio o indiretta con contagiati. Quello che in queste pagine risulta evidente è che il racconto social sul digitale può essere scisso secondo diverse articolazioni relative alle differenti sfere di prossimità non riducibili ad una sola dimensione, ma articolabili in un complesso di concomitanti influenze alle quali in questo studio abbiamo provato a dare forma legandole alle diverse geografie che producono tra COVID-Spread, COVID-Measures e COVID-Issues. I risvolti empirici del nostro lavoro ci portano di fronte ad una sorte di paradosso: l'emergenza che, anche se in tempi tendenzialmente differenti, ha colpito in modo quasi uniforme il nostro Paese (e l'intera comunità mondiale) ha prodotto esiti molto differenti sui territori. Al netto della rappresentazione mediatica dell'Italia «unita contro il nemico Coronavirus» e degli Italiani «uniti ma distanti» le evidenze emerse ci suggeriscono una "realtà" molto più sfaccettata e meno lineare rispetto a ciò che il senso comune ci ha portato a immaginare. Le esperienze delle persone durante la crisi sanitaria sono state condizio- 
nate dalle situazioni contingenti sui loro territori, in termini di impatto epidemiologico e normativo. Questo riporta il discorso su un piano di metodo e ci invita a ragionare sull'effettiva opportunità di ripensare la ricerca sociale in ambiente digitale: l'ancoraggio spaziale del dato può essere un tentativo per accrescere le potenzialità e smussare i limiti dei digital methods.

\section{BIBLIOGRAFIA}

Balzola A. (2020), L'educazione a distanza alla luce e all'ombra della pandemia, in «Mediascapes journal», 15: 203-210.

Barnes S. J., Diaz, M., Arnaboldi, M. (2020), Understanding Panic Buying During COVID-19: A Text Analytics Approach, in «Expert Systems with Applications», 169: 114360.

Bashar M. A., Nayak R., Balasubramaniam T. (2020), Topic, Sentiment and Impact Analysis: COVID19 Information Seeking on Social Media, in https://arxiv.org/abs/2008.12435

Battistelli F., Galantino M. G. (2020), Sociologia e politica del coronavirus, Milano: FrancoAngeli.

Bonacini L., Gallo G., Scicchitano S. (2020), Working from home and income inequality: risks of a 'new normal'with COVID-19, in «Journal of Population Economics», 34, 1: 303-360.

Campagna M., Floris R., Massa P. (2016), Le informazioni geografiche dei social network (SMGI) a supporto della pianificazione del turismo. L'esempio di Cagliari, in «Atti della XX Conferenza Nazionale Asita», Vicenza.

Capone V., Caso D., Donizzetti A. R., Procentese F. (2020), University student mental well-being during COVID-19 outbreak: What are the relationships between information seeking, perceived risk and personal resources related to the academic context?, in «Sustainability», 12, 17: 7039.

Carelli P., Vittadini, N. (2020), Social-virus. Piattaforme, istituzioni e hashtag durante la pandemia, in M. Sala e M. Scaglioni, L>altro virus. Comunicazione e disinformazione al tempo del COVID-19, Milano: Vita e Pensiero.

Cerrocchi L. (2020), L'adattamento a distanza della didattica (e dell'educazione) tra università, scuola e agenzie educative extra-scolastiche. L'attività del DESU Unimore, http://www.focus.unimore.it/attivita-desu-distanza/.

Chang, C. H., Monselise, M., \& Yang, C. C. (2021), What Are People Concerned About During the Pandemic? Detecting Evolving Topics about COVID-19 from Twitter, in «Journal of Healthcare Informatics Research», 5, 1: 70-97.

Chew, C., Eysenbach, G. (2010), Pandemics in the age of Twitter: content analysis of Tweets during the 2009 H1N1 outbreak, in «PloS one», 5, 11: e14118.

Consiglio S., Gambardella D., Serpieri R., Rebeggiani E., Grimaldi E., Pirone E., Lumino E. (2020), University teaching in the digital transition La didattica universitaria nella transizione al digitale, in «QTimes Journal of Education Technology and Social Studies», 3: 185-197.

Cusinato M., Iannattone S., Spoto A., Poli M., Moretti C., Gatta M., Miscioscia, M. (2020), Stress, Resilience, and Well-Being in Italian Children and Their Parents during the COVID-19 Pandemic, in «International Journal of Environmental Research and Public Health», 17, 22: 8297.

De Falco C., Crescentini N., Ferracci M. (2021), The spatial dimension in social media analysis. Theoretical and methodological characteristics, in G. Punziano e A. Delli Paoli, Handbook of Research on Advanced Research Methodologies for a Digital Society, Hershey: IGI Global.

De Santis E., Martino A., Rizzi, A. (2020), An Infoveillance System for Detecting and Tracking Relevant Topics From Italian Tweets During the COVID-19 in «Event in IEEE Access», 17, 8: 132527-132538.

DPCM 18 Ottobre 2020 https://www.trovanorme.salute.gov.it/norme/dettaglioAtto?id=76753

DPCM 3 Novembre 2020 https://www.trovanorme.salute.gov.it/norme/dettaglioAtto?id=76993

DPCM 4 Dicembre 2020 http://www.salute.gov.it/portale/nuovocoronavirus/dettaglioNotizieNuovoCoronavirus. jsp?lingua $=$ italiano $\&$ menu $=$ notizie $\& \mathrm{p}=$ dalministero $\& \mathrm{id}=5207$

Drylie-Carey L., Sánchez-Castillo S., Galán-Cubillo E. (2020), European leaders unmasked: COVID-19 communication strategy through Twitter, in «El Profesional de la Información», 29, 5. 
Essam, B. A., Abdo, M. S. (2020), How Do Arab Tweeters Perceive the COVID-19 Pandemic?, in «Journal of psycholinguistic research $\gg, 50,3: 507-521$.

Falcone R., Sapienza A. (2020), How COVID-19 Changed the Information Needs of Italian Citizens, in «International Journal of Environmental Research and Public Health», 17, 19: 507-521.

Figari F., Fiorio C., Gandullia L., Montorsi C. (2020), La resilienza del sistema italiano di protezione sociale all'inizio della crisi COVID-19: evidenze territoriali, in «Politica economica», 36, 1: 3-33.

Gaggi O., Kolasinska A. B., Mirri S., Prandi, C. (2020), The new classmate: an exploration of how COVID-19 affected primary schools activities in Italy, in «Proceedings of the 6th EAI International Conference on Smart Objects and Technologies for Social Good».

Germani A., Buratta L., Delvecchio E., Mazzeschi C. (2020), Emerging Adults and COVID-19: The Role of Individualism-Collectivism on Perceived Risks and Psychological Maladjustment, in «International Journal of Environmental Research and Public Health», 17, 10: 3497.

Giancola O., Piromalli L. (2020), Apprendimenti a distanza a più velocità. L'impatto del COVID-19 sul sistema educativo italiano, in «Scuola democratica $»$.

Graffigna G., Bosio C., Savarese M., Barello M., Barello S. (2020), \# I-Am-Engaged”: Conceptualization and First Implementation of a Multi-Actor Participatory, Co-designed Social Media Campaign to Raise Italians Citizens' Engagement in Preventing the Spread of COVID-19 Virus, in «Frontiers in psychology», 11: 2428.

Halbwachs, M. (1918), La sociologia di Émile Durkheim, Milano: FrancoAngeli, 2018.

Istat (2020a), Decessi per il complesso delle cause. Periodo gennaio-settembre2020 (https://www.istat.it/it/ files//2020/03/Nota-informativa-dati-mortalita-al-30-settembre.pdf)

Istat (2020b), Una stagione mancata: impatto del COVID-19 sul turismo (https://istat.it/it/archivio/242017)

Istituto Superiore di Sanità (2020a), Epidemia COVID-19 Aggiornamento nazionale 22 dicembre 2020, https:// www.epicentro.iss.it/

Istituto Superiore di Sanità (2020b) Rapporto ISS COVID-19 n. 18/2020 - Raccomandazioni per la raccolta e analisi dei dati disaggregati per sesso relativi a incidenza, manifestazioni, risposta alle terapie e outcome dei pazienti COVID-19. Versione del 26 aprile 2020.

Lenzo V., Quattropani M. C., Musetti A., Zenesini C., Freda, M. F., Lemmo, D., Cattivelli, R. (2020), Resilience contributes to low emotional impact of the COVID-19 outbreak among the general population in Italy, in «Frontiers in Psychology», 11: 3062.

Longley, P. A., Adnan, M., Lansley, G. (2015), The geotemporal demographics of Twitter usage, in «Environment and Planning», 47, 2: 465-484.

Manella, G. (2009), L'attualità della scuola ecologica di Chicago, in «Sociologia Urbana e Rurale», 90: 129-146.

Mauss M. (1925), Saggio sul dono, Torino: Einaudi, 2002.

Mazzara B.M., Mauceri S., Gavrila M. (2020), Gli italiani sotto l'onda anomala dell'emergenza. Il dominio dell'apprensione tra rischi percepiti e comportamenti responsabili, in C. Lombardo, e S. Mauceri, La società catastrofica. Vita e relazioni sociali ai tempi dell>emergenza COVID-19, Milano: FrancoAngeli.

Meschini F. (2020), Testi e conversazioni. Il racconto collettivo dei social network, in «Umanistica Digitale», 4, 8.

Moscadelli A., Albora G., Biamonte M. A., Giorgetti D., Innocenzio M., Paoli S., Bonaccorsi G. (2020), Fake news and COVID-19 in Italy: Results of a quantitative observational study, in «International Journal of Environmental Research and Public Health», 17, 16: 5850.

Noor S., Guo Y., Shah S. H. H., Fournier-Viger, P., Nawaz, M. S. (2020), Analysis of public reactions to the novel Coronavirus (COVID-19) outbreak on Twitter, in «Kybernetes».

Nurwidyantoro, A., Winarko, E. (2013), Event detection in social media: A survey, in «International Conference on ICT for Smart Society $\gg, 1-5$.

Procentese F., Capone V., Caso D., Donizzetti A. R., Gatti, F. (2020), Academic Community in the Face of Emergency Situations: Sense of Responsible Togetherness and Sense of Belonging as Protective Factors against Academic Stress during COVID-19 Outbreak, in «Sustainability», 12, 22: 9718.

Punziano G., De Falco C.C., Trezza D. (2020), La Geografia delle percezioni degli Italiani sul COVID-19. Compren- 
dere la pandemia e i suoi effetti attraverso l'analisi spaziale dei Tweet, in «XLI Conferenza Scientifica Annuale Regioni tra sfide e opportunità inattese », Abstract Book, 304.

Rivieccio, B. A., Micheletti, A., Maffeo, M., Zignani, M., Comunian, A., Nicolussi, F., Salini, S., Manzi, G., Auxilia, F., Giudici, M., Naldi, G., Gaito, S., Castaldi, S., Biganzoli, E. (2021), CoViD-19, learning from the past: A wavelet and cross-correlation analysis of the epidemic dynamics looking to emergency calls and Twitter trends in Italian Lombardy region, in «PloS one», 16, 2, e0247854.

Rogers, R. (2013), Digital methods, Cambridge: MIT press.

Rolandi E., Vaccaro R., Abbondanza S., Casanova G., Pettinato L., Colombo M., Guaita, A. (2020). Loneliness and Social Engagement in Older Adults Based in Lombardy during the COVID-19 Lockdown: The Long-Term Effects of a Course on Social Networking Sites Use, in «International journal of environmental research and public health», 17, 21: 7912.

Roma P., Monaro M., Colasanti M., Ricci E., Biondi S., Di Domenico A., Mazza C. (2020), A 2-Month Follow-Up Study of Psychological Distress among Italian People during the COVID-19 Lockdown, in «International Journal of Environmental Research and Public Health», 17, 21: 8180.

Rzeszewski M. (2018), Geosocial capta in geographical research-a critical analysis, in «Cartography and Geographic Information Science», 45, 1: 18-30.

Saire Chire J.E., Cruz Oblitas J.F. (2020), Study of coronavirus impact on parisian population from april to june using twitter and text mining approach, in «International Computer Symposium (ICS)»: 242-246.

Sunstein C. (2007), Republic.com 2.0, Princeton: Princeton University Press.

Suratnoaji, C., Nurhadi, N., Arianto, I. D. (2020), Public Opinion on Lockdown (PSBB) Policy in Overcoming COVID-19 Pandemic in Indonesia: Analysis Based on Big Data Twitter, in «Asian Journal for Public Opinion Research», 8, 3: 393-406.

Sutko, D.M., de Souza e Silva, A. (2011), Location-aware mobile media and urban sociability, in «New Media \& Society», 13, 5: 807-823.

Zajczyk, F. (1991), La conoscenza sociale del territorio: Fonti e qualità dei dati, Milano: FrancoAngeli.

Zhao, N., Cao, G. (2017), Quantifying and visualizing language diversity of Hong Kong using Twitter, in «Environment and Planning A: Economy and Space», 49, 12: 2698-2701. 